This item was submitted to Loughborough's Research Repository by the author.

Items in Figshare are protected by copyright, with all rights reserved, unless otherwise indicated.

\title{
Large eddy simulation of isothermal turbulent swirling jets
}

PLEASE CITE THE PUBLISHED VERSION

PUBLISHER

(C) Taylor \& Francis

VERSION

AM (Accepted Manuscript)

LICENCE

CC BY-NC-ND 4.0

REPOSITORY RECORD

Malalasekera, W., Salah S. Ibrahim, K.K.J. Ranga-Dinesh, and M.P. Kirkpatrick. 2019. "Large Eddy Simulation of Isothermal Turbulent Swirling Jets". figshare. https://hdl.handle.net/2134/5578. 
This item was submitted to Loughborough's Institutional Repository (https://dspace.lboro.ac.uk/) by the author and is made available under the following Creative Commons Licence conditions.

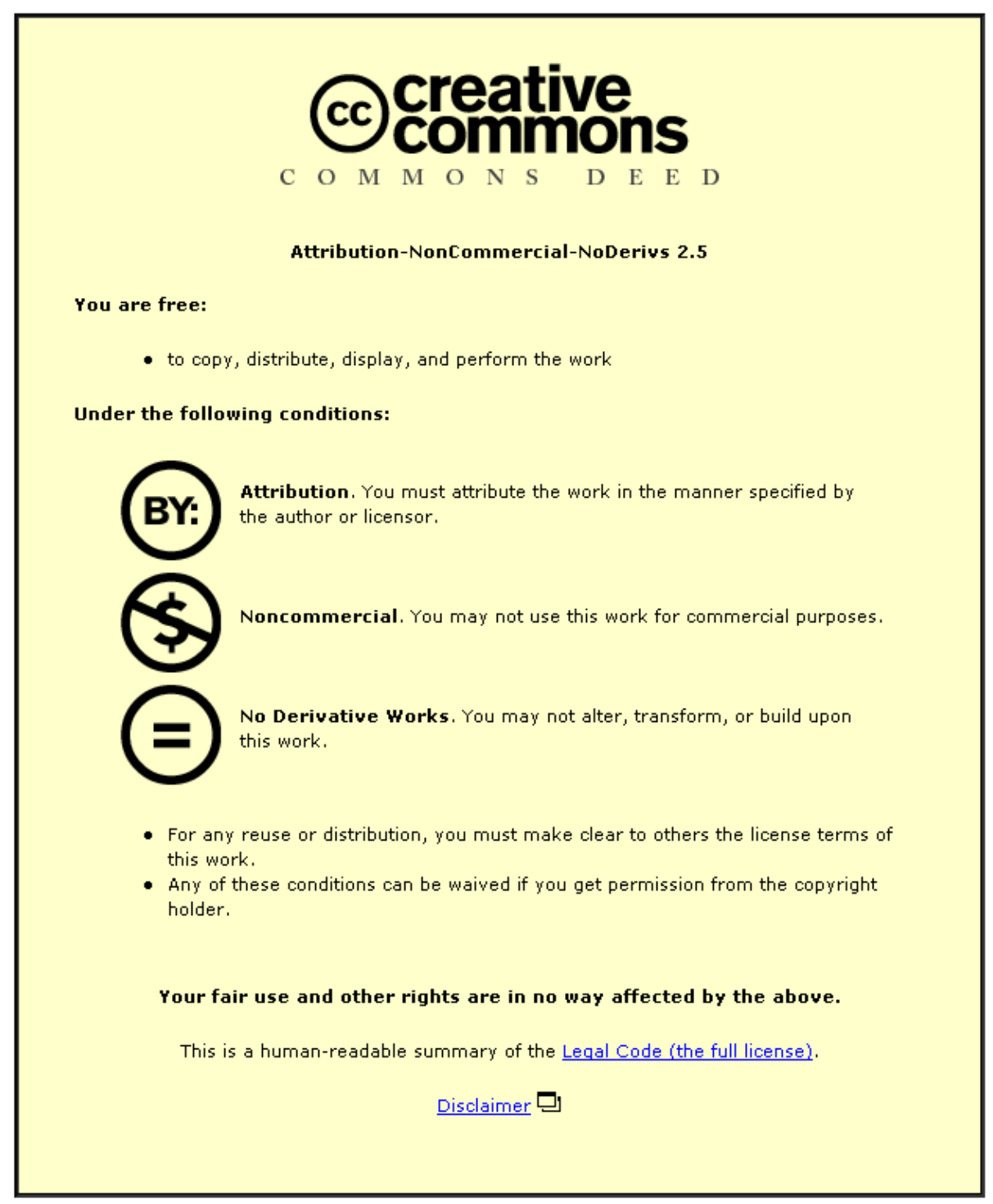

For the full text of this licence, please go to: http://creativecommons.org/licenses/by-nc-nd/2.5/ 


\section{LARGE EDDY SIMULATION OF ISOTHERMAL TURBULENT SWIRLING JETS}

W.Malalasekera ${ }^{1, *}$, S.S.Ibrahim ${ }^{2}$, K.K.J.Ranga Dinesh ${ }^{1}$ and M.P.Kirkpatrick ${ }^{3}$

1. Wolfson School of Mechanical and Manufacturing Engineering, Loughborough University, Loughborough, Leicestershire, LE11 3TU, UK.

2. Department of Aeronautics and Automotive Engineering, Loughborough University, Loughborough, Leicestershire, LE11 3TU, UK.

3. School of Aerospace, Mechanical and Mechatronic Engineering Building J07, Room S409, The University of Sydney

NSW 2006 Australia

* Corresponding author, W.Malalasekera@lboro.ac.uk

Submitted to Comb. Sci Tech, November 2005 


\title{
LARGE EDDY SIMULATION OF ISOTHERMAL TURBULENT SWIRLING JETS
}

\author{
W.Malalasekera ${ }^{1}$, S.S.Ibrahim ${ }^{2}$, K.K.J. Ranga Dinesh ${ }^{1}$ and M.P.Kirkpatrick ${ }^{3}$ \\ 1. Wolfson School of Mechanical and Manufacturing Engineering, \\ Loughborough University, Loughborough, Leicestershire, LE11 3TU UK. \\ 2. Department of Aeronautics and Automotive Engineering, \\ Loughborough University, Loughborough, Leicestershire, LE11 3TU UK. \\ 3. School of Aerospace, Mechanical and Mechatronic Engineering \\ Building J07, Room S409, The University of Sydney, NSW 2006 Australia.
}

Keywords: Large eddy simulation (LES), Swirl flow, Non-reacting, Turbulence

\begin{abstract}
Swirl stabilized flames are common in many engineering applications and modelling of such flames are particularly difficult due to their recirculation and vortex characteristics. Most classical approaches such as Reynolds averaged Navier-Stokes (RANS) models, which work very well in other situations, fail to perform well in high recirculation swirling flows. Large Eddy Simulation (LES) offers the possibility of improving calculations of such flows. This paper is concerned with the application of the LES technique to turbulent isothermal swirling flows. The aim is to improve our understanding of the flow physics and turbulence structure of unconfined swirling flows and examine the capability of LES to predict the formation of vortex breakdown in recirculation zones. In this study, a recently developed LES code has been applied to the prediction of isothermal swirling flows experimentally studied by $\mathrm{Al}$ Abdeli and Masri (2003). The filtered Navier-Stokes equations are closed using the Smagorinsky eddy viscosity model with localized dynamic procedure of Piomelli and Liu (1995). Advanced numerical schemes with finite volume formulation on non-uniform Cartesian grids are employed for discretization of the conservation equations. Three different test cases have been investigated here covering a range of swirl numbers and stream wise annular velocities. The cases considered have swirl numbers ranging from 0 to 1.59 and Reynolds numbers from 32400 to 59000 . With suitable inflow, outflow boundary conditions and sufficient grid resolutions the LES calculations found to be in good agreement with experimental data. It has been observed that the onset of downstream recirculation and vortex breakdown does not depend on the attainment of high swirl number alone. It appears that the bubble type vortex breakdown is achieved in the flow with a lower rather than higher swirl number. The axial momentum of the swirling annulus plays an important role in the onset of vortex breakdown. The combination of lower swirl number and higher axial velocity of the primary annulus leads to establish the downstream central recirculation zone (VB). These features have been successfully reproduced by LES calculations. For all the cases considered here LES calculations were successful in predicting observed recirculation zones and generally showed reasonably good agreement with experimentally measured mean velocities, their rms fluctuations and Reynolds shear stresses.
\end{abstract}




\section{NOMENCLATURE}

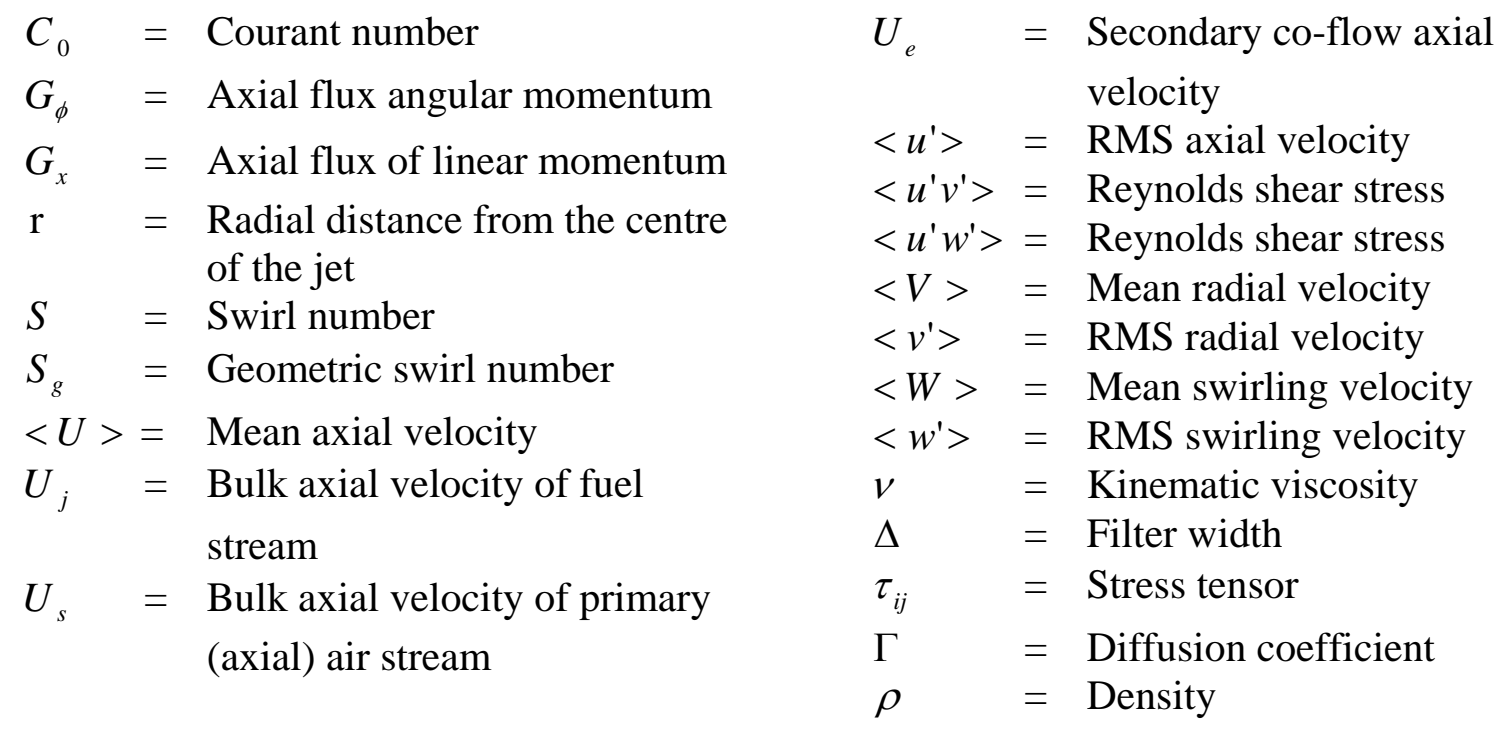

\section{INTRODUCTION}

Swirl is commonly used in many engineering applications of combustion to control essential characteristics of high intensity turbulent flows and to stabilise flames. Introduction of swirl has the capability to generate strong recirculation zones, which helps to improve the whole mechanism of the mixing process. Swirl can reduce the flame length by producing higher rates of entrainment and fast mixing particularly in the shear layer region, which improve flame stability, reduce emissions and as a result the burner can be minimized in size and has an extended life (Syred and Beér, 1974). In swirling jets and flames, sufficiently strong swirl will produce a maximum pressure gradient in the direction of the flow, which in turn produces a reversal of the flow or vortex breakdown. Depending on the degree of swirl, furnace and burner geometry, different recirculation patterns and vortex breakdown regimes can be achieved and these can be controlled by adjusting the degree of swirl to promote better mixing, flame stabilisation and minimise pollutant formation (Sloan et al., 1986).

In the literature, different flow configurations have been experimentally investigated to study the formation of recirculation zones and the vortex breakdown process. Theoretical studies have been carried out to analyse the instabilities and onset of vortex breakdown for reacting and non-reacting swirling flows and it has been reported that the influence of swirl depends on different flow parameters such as inflow velocity profiles, Reynolds number, level of swirl and geometrical configuration. Many situations such as combustion related applications (Syred and Beér, 1974, Gupta et al., 1977, Weber et al., 1990, Masri et al., 2004), confined 
pipe situations (Nathan et al. 1998, Ahmed, 1998) vortex whistles and model cyclone separators (Kitoh, 1991) have been studied in the literature. A number of good reviews and a body of literature exist on these topics ranging from vortex breakdown to instabilities of swirling flows (see for example Sarpkaya, 1971, Syred and Beér, 1974, Escudier and Zehnder, 1982, Escudier, 1988, Lucca-Negro and O’Doherty, 2001, Novak and Sarpkaya, 2001). Numerical modelling has also been used as a tool to understand the fundamental flow physics of laminar and turbulent swirling flows. The numerical prediction of turbulent swirling flows is a challenging subject due to anisotropic turbulence structure in recirculation zones. An extensive review of the modelling work on swirling flows has been reported by Sloan et al. (1986), which describe the difficulties and complexities associated with CFD approaches to swirling flow calculations.

The majority of current methods to model turbulent swirling flames and practical combustion systems are based on Reynolds averaged Navier-Stokes (RANS) equations accompanying different turbulence models. The review by Slone et al. (1986) summarises many RANS type swirling flow calculations with different turbulence model options and concludes that the performance of the $k-\varepsilon$ model is generally not very good in the vicinity of recirculation zones. The review also describes various advanced turbulence model options which appear to show some improvements and note that swirling flows exhibits highly three-dimensional structures therefore steady-state axi-symmetric transport equations are incapable of reproducing complex swirling flows. Weber et al. (1990) have assessed three turbulence models, namely, the standard two-equations eddy viscosity $k-\varepsilon$ model, Reynolds stress model (RSM) and algebraic Reynolds stress model (ASM) to simulate confined swirling flows. It was observed that neither the generation of turbulence nor the distribution of tangential momentum was correctly predicted by the $k-\varepsilon$ model. In contrast the Reynolds stress model and algebraic Reynolds stress model performance were better and produced reasonable agreement with experimental data for certain confined swirl cases but for certain other cases none of the turbulence models was able to predict the correct reverse flow when compared with measured data. In general RANS models are primarily suitable to calculate stationary flows with non-gradient transport and it is difficult to capture the unsteady nature of the large-scale flow structures typically found in turbulent swirling flows. Guo et al. (2001) have used a three-dimensional unsteady RANS approach with the $k-\varepsilon$ model to compute behaviour of unconfined swirling flows in an axisymmetric sudden expansion. The study has successfully predicted the processing phenomena of the swirling flow configuration considered in their paper but no detailed comparison of velocity or Reynolds stresses have 
been reported to assess fine details of the simulation. However the study confirms that to achieve any degree of success, RANS type computations have to be transient and threedimensional.

Large eddy simulation (LES) in contrast is a powerful and effective tool for handling largescale turbulent motions as the method is based on time resolved three-dimensional unsteady large-scale turbulent motions. In LES only the small scale eddies which have more universal behaviour and contain less turbulent kinetic energy are modelled. With adequate spatial and temporal resolution the method is capable of capturing large-scale dynamic behaviour in flows. Large eddy simulation technique as a tool for the simulation of swirling flow fields in both reacting and a non-reacting cases has emerged only in the 1990s and hence a relatively new field. LES has been applied to variety of swirling applications including combustion such as aircraft engine combustion (Di Mare et al. 2004, Kim el al. 1999), dynamics of swirling premixed and spray flames (Sankaran and Menon, 2002) and combustion instabilities (Wall and Moin, 2005). Recently the application of LES to real combustion devices has gained increased popularity due to its potential ability to capture detailed flow and mixing fields and the availability of computing power to perform large calculations. For example, the LES simulation of a section of the modern Pratt and Whitney gas turbine combustor by Mahesh et al. (2005) has shown encouraging results for the flow and mixing field.

One of the main advantages of LES applications is, in swirl flows it can capture oscillatory motions such as pressing vortex core (PVC) seen in experiments. Pierce and Moin (1998) for example showed promising agreement between LES and experimental data for a low swirl number case and Wang et al. (2004) have shown successful comparison between LES predictions and experimental measurements operating under different conditions in confined turbulent swirling flows. More recently several LES simulations have also been carried out for laboratory scale bluff body stabilized flames, Raman and Pitsch, (2005), Kempf et al. (2006), which are not as complex as swirling flows but contain similar recirculation zones near the bluff body. The predicted results show very good agreement with experimental data.

In this paper, the LES simulation technique has been applied to simulate a well-defined swirling flow configuration where reliable and accurate data are available. The configuration is an unconfined, non-reacting swirling flow burner. It is widely known as the Sydney swirl burner, experimentally investigated by Al-Abdeli and Masri (2003). For this configuration detailed experimental measurements are available for the flow field using the laser Doppler 
velocimetry (LDV). The LES simulations have been carried out using different grid resolutions and appropriate input and outflow boundary conditions. The calculated results are compared with experimental data for three different test cases ranging from no swirl to very high swirl. Comparisons between calculated and measured data are made for the mean velocities, rms fluctuations and Reynolds stresses components. The Smagorinsky (1963) eddy viscosity model with the localized dynamic procedure of Piomelli and Liu (1995) is used to model the sub-grid scale (SGS) turbulence. This is a first step in a series of calculations, where the focus is on the fluid dynamical aspects of the mixing process in the recirculation zones. The ultimate aim of this work is to model both the flow and combustion characteristics of this swirl burner. The predictive capability of the flow physics of non-reacting swirling flows is considered in this paper before attempting to include SGS combustion models to simulate reacting swirling flames. In the following sections details of the experimental setup are described, followed by description of the mathematical model and details of the numerical computations. The results are presented for three different cases with zero swirl, moderate swirl and high swirl flow configurations.

\section{EXPERIMENTAL SETUP}

Schematic drawing of the swirl burner used in this investigation is shown in Figure 1. The burner consists of a 50mm cylindrical face bluff body with $3.6 \mathrm{~mm}$ diameter central fuel jet. Surrounding the cylindrical bluff body is a $60 \mathrm{~mm}$ diameter annulus machined down to $0.2 \mathrm{~mm}$ thickness at the exit plane. The centre of the jet is taken as the geometric centre-line of the flow where $r=0$ and $x=0$. The burner is housed in a secondary axial (co-flow) wind tunnel with square cross section and $130 \mathrm{~mm}$ sides. Swirl is introduced aerodynamically into the primary (axial) air stream at a distance 300mm upstream of the burner exit using tangential swirl ports as shown in Figure 1. An axial stream of air is also supplied through two opposed ports ahead of the tangential inlets. By changing the relative magnitude of the tangential and axial air flow rates the swirl number can be varied. Laser Doppler Velocimetry (LDV) technique has been used to measure the detailed characteristics of the flow field and for the non-reacting cases they include mean velocity, their rms fluctuations and Reynolds shear stresses. Further details of the experiments and data are available in Al-Abedeli and Masri (2003) and Masri et al. (2004).

\section{Swirl Number}


The swirl number is defined as the ratio between the axial flux of the swirl momentum, $G_{\phi}$ $\left(\mathrm{kgm}^{2} \mathrm{~s}^{-2}\right)$, to the axial flux of the axial momentum $G_{x}\left(\mathrm{kgm}^{2} \mathrm{~s}^{-2}\right)$ multiplied by a characteristic length $R(\mathrm{~m})$. Here we take the radius of the swirl annulus as the characteristic radius. The swirl number is given by:

$$
S=\frac{G_{\phi}}{R G_{x}}=\frac{\int_{0}^{R} U W r^{2} d r}{R \int_{0}^{R} U^{2} r d r}
$$

where $U(\mathrm{~m} / \mathrm{s})$ and $W(\mathrm{~m} / \mathrm{s})$ are the mean axial and tangential velocities, respectively, at the exit plane of the swirl generator. Four independent parameters are used to describe the swirl flow conditions in this experimental burner configuration. These parameters are, the bulk axial velocity of fuel stream, $U_{j}$, bulk axial and tangential velocities of the primary (air) stream, $U_{s}$ and $W_{s}$ respectively and the mean co-flow velocity of the secondary (air) stream in the wind tunnel, $U_{e}$. The later stream is necessary to provide well-defined outer boundary conditions. The Reynolds number of the swirl annulus air stream is defined in terms of the primary (bulk) axial velocity $\left(U_{s}\right)$, radius of the burner annulus $\left(r_{s}\right)$ and the kinematic viscosity of air $v$ such that $\operatorname{Re}_{s}=U_{s} r_{s} / v$. In the experimental measurements a quantitative representation of the swirl intensity has been introduced by using the geometric swirl number $\left(S_{g}\right)$, which is expressed as the ratio of integrated (bulk) tangential velocity to primary axial air velocities $\left(W_{s} / U_{s}\right)$. It is found that the actual swirl number, $S$ defined by eq. (1) is linearly proportional to the geometric swirl number $S_{g}$ (Al-Abdeli, 2003). The following three cases summarised in Table 1 are considered in our simulations. The control parameters shown in Table 1 and case numbers have been obtained from Al-Abdeli and Masri (2003).

\begin{tabular}{|c|c|c|c|c|c|c|}
\hline Flow case & $U_{s}(\mathrm{~m} / \mathrm{s})$ & $W_{s}(\mathrm{~m} / \mathrm{s})$ & $U_{j}(\mathrm{~m} / \mathrm{s})$ & $U_{e}(\mathrm{~m} / \mathrm{s})$ & $S_{g}$ & $\operatorname{Re}_{s}$ \\
\hline N21S000 & 21.1 & 0 & 61 & 20 & 0 & 41900 \\
\hline N29S054 & 29.7 & 16 & 66 & 20 & 0.54 & 59000 \\
\hline N16S159 & 16.3 & 25.9 & 66 & 20 & 1.59 & 32400 \\
\hline
\end{tabular}

Table 1: Physical flow parameters used in the study 


\section{MATHEMATICAL MODELING}

\section{The Governing Equations}

In LES the large-scale energy containing flow are resolved numerically while the small, unresolved scales and their interactions with the large scales are modelled. The grid-filtering operator known as the spatial filter is applied to decompose the resolved and sub-grid scales in the computational domain. The application of a spatial filter $G$ to a function $f$ is defined as

$$
\bar{f}(x)=\int_{\Omega} f\left(x^{\prime}\right) G\left(x-x^{\prime}, \bar{\Delta}(x)\right) d x^{\prime}
$$

where $\bar{\Delta}$ is the characteristic width of the filter and $x$ is the position vector in a Cartesian coordinate system. Commonly used filters are Fourier, Gaussian and box filters. A box filter naturally fits into the finite volume formulation and here we apply a box filter of width

$$
\bar{\Delta}=2(\Delta x \Delta y \Delta z)^{1 / 3}
$$

to the equations. Where $\Delta x, \Delta y$ and $\Delta z$ refer to the width of the finite volume cell in three coordinate directions. Applying the spatial box filter to incompressible Navier-Stokes equations, we obtain the filtered continuity and momentum equations for the large-scale motion as follows.

Conservation of mass

$$
\frac{\partial \bar{u}_{j}}{\partial x_{j}}=0
$$

Conservation of momentum

$$
\frac{\partial \bar{u}_{i}}{\partial t}+\frac{\partial\left(\bar{u}_{i} \bar{u}_{j}\right)}{\partial x_{j}}=-\frac{1}{\rho} \frac{\partial \bar{P}}{\partial x_{i}}+\frac{\partial\left(2 v \bar{S}_{i j}\right)}{\partial x_{j}}+\frac{\partial\left(\tau_{i j}\right)}{\partial x_{j}}
$$

Where the strain rate tensor, $S_{i j}=\frac{1}{2}\left(\frac{\partial \bar{u}_{i}}{\partial x_{j}}+\frac{\partial \bar{u}_{j}}{\partial x_{i}}\right)$.

The last term of equation (5) represents the sub-grid scale (SGS) contribution to the momentum and it is known as the SGS stress tensor. Hence subsequent modelling is required for $\tau_{i j}=\left(\bar{u}_{i} u_{j}-\bar{u}_{i} \bar{u}_{j}\right)$ to close the system of equations. 


\section{SGS Turbulence Modelling}

The Smagorinsky (1963) eddy viscosity model is used here to model the SGS stress tensor $\tau_{i j}=\left(\bar{u}_{i} u_{j}-\bar{u}_{i} \bar{u}_{j}\right)$ such that

$$
\tau_{i j}-\frac{1}{3} \delta_{i j} \tau_{k k}=-2 v_{s g s} \bar{S}_{i j}
$$

Here the eddy viscosity $v_{s g s}$ is a function of the filter size and strain rate

$$
v_{s g s}=C_{s} \bar{\Delta}^{2}|\bar{S}|
$$

where $C_{s}$ is a Smagorinsky (1963) model parameter and $|\bar{S}|=\left(2 \bar{S}_{i j} \bar{S}_{i j}\right)^{\frac{1}{2}}$.

The localized dynamic procedure of Piomelli and Liu (1995) has been used to calculate the model parameter $C_{s}$, which appears in equation (7).

\section{COMPUTATIONAL DETAILS}

\section{Numerical Discretisation:}

The large eddy simulation code PUFFIN originally developed by Kirkpatrick (2002) is used to perform all calculations presented here. The equations are discretised in space by using a finite volume formulation on a non-uniform, staggered, Cartesian grid. A second order central difference approximation is used for all terms in the momentum equations and pressure correction equation. The solution is advanced in time by using the fractional step method. First the momentum equations are integrated using a third order hybrid Adam-Bashforth/ Adam-Moulton scheme to give an approximate solution for the velocity field. Then the mass conservation is enforced through a pressure correction step in which the approximate velocity field is projected onto a subspace of divergence free velocity fields. The pressure correction method of Van Kan (1986) and Bell et al. (1989) is used in the present calculations. Gresho (1990) has shown analytically that this method is second order accurate in time. The time step is varied to ensure that the Courant number $C_{o}=\Delta t u_{i} / \Delta x_{i}$ remain approximately constant. Where $\Delta x_{i}$ is the cell width, $\Delta t$ is the time step and $u_{i}$ is the velocity components in the $x_{i}$ direction. The solution is advanced with a time stepping corresponding to Courant number in the range of $C_{o}=0.3$ to 0.6 . Further details of the second order time stepping schemes and fractional step method used here can be found in Kirkpatrick (2003a, b).

The equations, discretised as described above, are solved using a linear equation solver. Here a Bi-Conjugate Gradient Stabilized (BiCGStab) solver with a Modified Strongly Implicit 
(MSI) preconditioner is used. The momentum residual error is typically of the order $10^{-5}$ per time step and the mass conservation error is of the order of $10^{-8}$.

\section{Boundary conditions:}

Correct representation of the inflow profiles and turbulence levels is an important issue for successful simulation of swirling flows. This has been widely discussed in the recent Turbulent Non-premixed Flames workshop (TNF8, 2006) and there is a general agreement that correct mean and fluctuations at the inlet is required for large eddy simulations to produce accurate results. There are several methods available to introduce turbulent inlet boundary conditions. In this study mean velocity distributions for the fuel and air annulus are specified using power law velocity profiles. This approach has been previously used by Masri et al. (2000) in their two-dimensional, axisymmetric, pdf computation of combustion in this burner configuration.

In the fuel jet, $\langle V>=0,\langle W>=0$, and the mean axial velocity is specified by a power law of the form

$$
<U>=1.218 U_{j}\left(1-\frac{|y|}{\delta}\right)^{1 / 7}
$$

where $U_{j}$ is the bulk velocity, $y$ is the radial distance from the jet centre line and $\delta=1.01 R_{j}, R_{j}$ is the fuel jet radius $(1.8 \mathrm{~mm})$. The factor 1.01 is included in the expression for $\delta$ to ensure that velocity gradients are finite at the walls. The same equation (eqn. 9) is used for the swirling air stream with $U_{j}$ replaced by bulk axial velocity $U_{s}$ and bulk tangential velocity $W_{s}$ and $y$ being the radial distance from the centre of the annulus and $\delta=1.01$ times half width of the annulus. For the secondary air stream the measured velocity $U_{e}$ is specified and $\langle V\rangle=\langle W\rangle=0$. Following the approach of Masri et al. (2000) fluctuations are added to the mean velocity profiles such that the inflow has the correct turbulence kinetic energy levels obtained in the experimental data.

\section{Statistics:}

The time averaged mean velocity components and their mean fluctuating values in the axial, radial and azimuthal directions are obtained by time averaging the transient flow variables obtained from LES results, i.e. 


$$
<\bar{\phi}>=\frac{1}{N_{t}} \sum_{n=1}^{N_{t}} \bar{\phi}^{n}, \quad \bar{\phi}_{r m s}=\sqrt{\frac{1}{N_{t}} \sum_{n=1}^{N_{t}}\left(\bar{\phi}^{n}-<\bar{\phi}>\right)^{2}}
$$

Where $N_{t}$ represents the number of samples. To remove the non-physical artefacts of the initialisation, the simulation should evolve for a sufficiently long time before gathering any statistical results. This allows the flow field to fully develop and initial transients to exit the computational domain. The samples are only taken after the flow filed has fully developed. In this study to obtain statistically stationary results, time averaging of the primitive variables was performed after 12 flow-through-times $(\tau)$, which is defined here as the time for a fluid element to propagate through the computational domain, i.e. $\tau=L / U, L$ and $U$ are axial length of the computational domain and inlet bulk axial velocity respectively. Two nonconsecutive sampling periods yielded similar results indicating that the statistics were sufficiently converged.

The calculations were performed on a Pentium 4, personal computer with 4GB RAM and $3 \mathrm{GHz}$ processor running under Linux operating system. A typical run takes 10-17 days depending on the inlet parameters and the number of flow passes. Table 2 summarise typical run times in days for each case considered.

\begin{tabular}{|c|c|c|c|c|c|c|}
\hline Flow case & Grid Type & $N_{x}$ & $N_{y}$ & $N_{z}$ & Time (ms) & Cost days \\
\hline N21S000 & Grid1 & 100 & 100 & 100 & 150 & 10 \\
\hline N29S054 & Grid1 & 100 & 100 & 100 & 150 & 12 \\
\hline N16S159 & Grid1 & 100 & 100 & 100 & 200 & 14 \\
\hline N16S159 & Grid2 & 120 & 120 & 100 & 300 & 17 \\
\hline
\end{tabular}

Table 2: Computational parameters used in the study. $N_{x}, N_{y}$ and $N_{z}$ are the number of nodes in the $x, y$ and $z$ directions respectively.

\section{Grid Dependency Tests:}

The computational domain used in the present calculation was 130x130x250 mm. Two grids were employed to conduct a grid sensitivity analysis of LES. Grid 1 consisted of $100 \times 100 \times 100$ cells in $x, y$ and $z$ directions respectively giving a total of one million grid points. A second grid, Grid 2 that is finer than Grid 1 was used with a total of 1.44 million grid points and consisted of $120 \times 120 \times 100$ cells in $x, y$ and $z$ directions respectively. The axial resolution was kept the same for both grids due to computer resource limitations. For Grid 1, grid lines in $x$ and $y$ directions used an expansion ratio of $\gamma_{x y}=\Delta x(i) / \Delta x(i-1)=1.08$ 
and an expansion ratio of $\gamma_{z}=1.07$ was used in the z-direction. The expansion ratio for Grid 2 was $\gamma_{x y}=1.06$ in the $\mathrm{x}$ - and $\mathrm{y}$-directions and $\gamma_{z}=1.07$ in the z-direction.

The case N16S159 with a swirl number of 1.59, which has the highest swirl number among the cases considered in this study was used as the test case for grid sensitivity analysis. It is not possible to define classical grid independence with implicit filtering when filter width directly affected by the grid (Kempf et al., 2006). However a refined grid can minimize both numerical and modelling errors through better resolution. LES results obtained here using the above two grids are compared with experimental measurements in Figure 2 where the mean axial velocity and its rms fluctuations at two different axial locations are shown. Solid lines represent the Grid 1 results ( 1 million points), dashed lines represent the Grid 2 results (1.44 million grid points) and symbols represent experimental measurements. The comparisons between the experimental measurements and computed mean axial velocity and its rms fluctuations at $x / D=0.136,0.2$ are very good close to the burner axis and in good qualitative agreement at $r / R>1$. There is only a slight difference between grid 1 and grid 2 predictions in the mean axial velocity plots, in the outer shear layer of the bluff body stabilized recirculation zone. Overall, including other data (not presented here) it was observed that both grids give very similar results for mean, rms and Reynolds stresses for the case N16S159 and hence we have used grid 1 with 1 million grid points for all our LES simulations to keep the computational cost at an economical level, (see Table 2) allowing to compute all the nonreacting cases in this flame series.

\section{RESULTS AND DISCUSSION}

In this section the comparison of the LES computations and experimental measurements is discussed for three different test cases namely N21S000, N29S054 and N16S159 (as described in Table 1).

The predictive capability of the flow physics and structure of non-reacting swirling flows over a wide range of swirl numbers is essential to enable combustion models to simulate the swirling flames. This is because in reacting flows, the interaction between turbulence and chemistry becomes significant due to the existence of complex dynamical flow structure induced by swirl. Especially, introduction of swirl is intrinsically associated with the phenomenon of vortex breakdown (Lucca 2001), which adds another dimension to the 
complexity of the problem. The systematic validation of computation is therefore necessary to have a full understanding of the complex transient behaviour of swirling motion.

In the configuration considered here adequate swirl can induce two types of recirculation zones. One is known as the torroidal shaped recirculation zone, which stagnates above an obstruction such as a bluff body, placed in axial flows. Other recirculation zones, which can be formed further downstream, are attributed to vortex breakdown (Al-Abdeli and Masri 2003). The formation of a stagnation point or a region of reversed flow is essential to generate this kind of recirculation zones in a swirling flow.

Experimental results show that for the swirl test cases considered here the mean flow field exhibits one or more recirculation zones depending not only on the swirl number, but also on the Reynolds number of the primary annulus. The appearance of recirculation zones is very much a fluid dynamic feature, which depends on local flow parameters and vortex breakdown. The strength of which determine the level of turbulence. Successful prediction for such flow, therefore, depends on correct representation of the turbulent flow field and associated regions of swirl flows. These features represent a good challenge for the LES technique.

Results obtained using the LES model, explained above, have been compared with the corresponding experimental data for all three cases. The comparisons are presented and discussed in the following sections. For all presented results, except the steam-line plots, the axial position $x$ is normalized by the diameter of the bluff body $D=50 \mathrm{~mm}$ and the radial position $r$ is normalized by the radius of the bluff body $R=25 \mathrm{~mm}$ the radial position.

\section{Flow field and characteristic features of the non-swirling case (N21S000)}

Figure 3 shows the streamlines generated from the mean velocity field in the central $x-z$ plane of the non-swirl case (N21S00). The mean streamlines show a two-zone flow/turbulence structure. A typical torroidal shape recirculation zone is formed due to the sudden expansion at the bluff body wall. The central jet velocity is dominating the wake: there is no stagnation point located along the centreline. No lateral spread being imposed onto the central jet by the recirculation zone. The schematic streamlines of the two counter-rotating vortices associated with the central jet and co-flow can be seen in Figure 3. It can, also, be seen that the co-flow driven vortex is wider than the central jet affected vortex. Although both central jet and co-flow velocity are operating, the external airflow principally manages the 
recirculation zone structure. The stream line pattern also shows the formation of a neck zone above the recirculation zone. Two shear layers are observed, first between the central jet and the inner vortex and the second is formed between the outer edge of the outer-vortex and the co-flow.

Figures 4 shows the computed radial profiles of time averaged mean axial velocity compared with experimental data at different positions along the burner axis. The calculated mean axial velocity at $x / D \in\{0.074,0.2,0.4\}$ shows minor under prediction at the centreline as a result of an early break up of the central jet. Despite this small discrepancy, the predictions for the mean axial velocity are in good agreement with experimental measurements. The existence of negative mean axial velocity indicates the flow reversal, which generates the bluff body stabilized recirculation zone. It can be seen that the length of the bluff body wake is completely captured by the simulation. The recirculation zone is mainly created due to the deceleration of the axial velocity by the sudden expansion at the bluff body wall. It can be noted that the recirculation zone extends up to $x / D=0.8$ in the axial direction. This can be seen where the mean axial velocity exhibits some negative values at $x / D=0.8$ and becomes completely positive at the measurement location $x / D=1.4$ and remain positive after that for all down stream axial locations.

Figure 5 shows comparisons between calculated and measured values for the mean radial velocity component. Some notable discrepancies are evident at near burner locations. However, at $x / D=0.074$, correct trend is obtained with a slight over-prediction for the peak value. Some differences between calculated and measured results are observed at $x / D=0.2$ where experimental data show an unusual abrupt reversal of the radial velocity magnitude, which is not captured by the LES model. Data taken in an equivalent bluff body configuration (Dally et al. 1998) in which similar non-swirling experiments were conducted does not show such a discontinuity in radial velocity. This could be due to experimental difficulties in measuring radial velocities very near the exit of the swirl annulus. The predicted profile at this location captures the experimental data up to $r / R=1.0$ reasonably well. Further downstream the radial velocity is under predicted between the regions $r / R=0.2-1.0$ at locations $x / D \in\{0.4,0.6\}$. It can be seen that despite the upstream discrepancies, the agreement between predictions and experiments are good at most downstream axial locations. It is worth noting that the magnitude of the radial component is small compared to the axial velocity values hence the discrepancies are relatively small. 
Calculated results for the rms fluctuations of axial, $\left\langle u^{\prime}\right\rangle$, and radial, $\left\langle v^{\prime}\right\rangle$, velocity components are compared and presented in Figures 6 and 7. In agreement with the observed wake properties, results show high rms values for both axial and radial fluctuations in regions where the central jet is constrained by the recirculating flow. At most upstream locations the comparisons for the axial rms velocity are good (Figure 6). Sharp changes and peak values have been correctly predicted and calculations show good agreement up to $x / D=0.8$. Further downstream the predicted profiles of $\left\langle u^{\prime}\right\rangle$ show slight over-predictions. Comparison of rms radial velocity shown in Figure 7 shows some discrepancies with experimental data. However, rms of radial velocities are slightly over predicted between the region $r / R=0.5-1.0$ at the first five axial locations. Further down stream, the comparison shows slight over predictions. Given that absolute magnitudes of rms velocities are difficult to calculate, the model's overall ability to predict the peak in rms velocities and their trend appears to be good.

Comparisons of predicted and measured Reynolds shear stresses $\left\langle u^{\prime} v^{\prime}\right\rangle$ for this case are shown in Figure 8. The LES model found to capture the peaks and the changes of the stresses inside the recirculation region reasonably well. Although some under predictions can be seen in region between $r / R=0.0-0.7$ at the axial location $x / D \in\{1.4,2.0,2.5\}$. The predictions, however, produced better agreement with the experimental data in the near burner region.

In general, the LES model described in this paper, has been successful in predicting key flow features of the N21S000 case. The overall agreement for this case between measurements and calculations for mean velocities, rms fluctuations and Reynolds shear stress is seen to be good.

\section{Flow field and characteristic features of the medium-swirl case (N29S054):}

Here, the results for the medium swirl case, a swirl number of $S=0.54$ are presented and discussed in terms of comparison between the model predictions and experimental measurements.

The streamlines generated from the mean velocity field of the medium swirl case are shown in Figure 9. Recirculation patterns seen in this case are different to the patterns seen in Figure 3. Non-swirl flows stabilized on this burner have a single recirculation zone. With the addition of swirl, the flow field can exhibit more complex flow patterns involving the formation of a second recirculation zone (Al-Abdeli and Masri 2003). This is evident in 
Figure 9. In the upstream recirculation zone, two counter rotating vortices similar to the nonswirl case and another small vortex on top of the bluff body wall can be seen. Here the near burner flow features are completely different to the non-swirl case as the flow is now affected by the primary axial and swirling velocities. The size of eddies inside the upstream recirculation zone are relatively smaller than those formed in the non-swirl case. As seen in Figure 9, the swirl induced downstream recirculation zone stagnates near the central axis away from the burner surface at about $x=70 \mathrm{~mm}$. This centreline recirculation zone, which appears well away from the burner exit, is called the vortex breakdown (VB) bubble. It is interesting to note that present LES simulation has captured both types of recirculation zones and further comparison of mean data shown in figures and described below confirm the success of LES simulations in predicting recirculation and vortex breakdown.

Figure 10 shows the measured and computed mean axial velocity at different downstream positions. A positive to negative change in mean axial velocity indicates the development of recirculation regions. It can be seen that the first recirculation zone develops above the bluff body and stagnates at about $x / D=0.4$ from the burner exit plane and second the second recirculation zone develops further downstream.

As mention in the introduction, swirl is often used in combustors to increase chemical and thermal mixing rates and for flame stability. At sufficiently high level of swirl, the VortexBreakdown (VB) phenomenon is known to occur (Sarpkaya, 1971). VB is characterised by a flow reversal along the centreline and is a result of an adverse pressure gradient induced by the highly swirling flow. This is due to force imbalance between outward expulsions of fluid and the axial momentum (Escudier, 1988). The existence of second recirculation zone is called the vortex breakdown (VB) and in this case occurs at about $x / D=1.8$. As can be seen in Figure 10, the centreline velocity rapidly decays with increasing distance downstream. Data shows that between $1.1<x / D<2.1$, the centreline velocity become negative and the flow stagnate creating the bubble type VB zone at about $x / D=1.8$. Present LES predictions have correctly reproduced this phenomena except that the predicted centreline velocity shows slight under-predictions at $x / D=0.4$. Further downstream of the VB zone predictions show the correct trend but agreement is not as good as the near burner region. Overall it could be said that LES results for axial velocity predictions capture the correct changes in the flow field, recirculation and VB phenomena. The success of LES to predict the swirl flow features including VB phenomenon is very encouraging. 
The mean radial velocities are shown in Figure 11. Profiles at upstream locations capture the correct trend and sharp changes and the comparisons with data are reasonably good, especially the peaks at $x / D \in\{0.136,0.2\}$ have been correctly predicted. LES correctly predict the trend at $x / D=0.4$ and 0.6 but in the inner-region under-predicts peak values of radial velocity. Further downstream at $x / D \in\{1.4,2.0,2.5\}$ radial velocity is over predicted. However, data shows that at $x / D \in\{2.0,2.5\}$ the experimental mean radial velocity is about $-5 \mathrm{~m} / \mathrm{s}$ at the centreline. This means the flow has been constantly tilted towards one direction and there has been considerable asymmetry. The LES predictions, presented here, have been axi-symmetrically averaged in the calculation of mean values therefore a non zero value for the mean radial velocity is not expected at the centre line. The predictions therefore show zero radial velocity at the centre line at these locations (which is the correct mean). There are some concerns over experimental data for mean radial velocity at some axial positions, $x / D \in\{1.4,2.0,2.5\}$.

Comparison of predicted and measured swirl velocity $\langle W\rangle$ is shown in Figure 12 . The agreement is generally good. Predictions compares well at locations $x / D \in\{0.136,0.2,0.4,1.4\}$ capturing the sharp changes and peak values. At locations $x / D \in\{0.6,0.8\}$ the trend is correctly predicted but the peak values are under predicted. Further downstream calculations show slight over predictions with increasing radial distance but overall agreement is reasonably good.

Figures 13-15 show comparison of measured and computed rms fluctuations of axial, radial and swirling velocities. Figure 13 shows the rms of axial velocity, $\left\langle u^{\prime}\right\rangle$. In general agreement between the LES predictions and experiments are good. However, the centre line values of $\left\langle u^{\prime}\right\rangle$ show under predictions at all locations. The agreement for rms radial velocity (Figure 14) and rms swirling velocity (Figure 15) are also generally encouraging at all axial locations. The rms radial velocity is slightly over predicted at the outer shear layer of the upstream recirculation zone at $x / D \in\{0.136,0.2\}$. The same behaviour is observed for the rms swirling velocity as well (figure 15). Magnitudes and distribution of $\left\langle u^{\prime}\right\rangle,\left\langle v^{\prime}\right\rangle$ and $\left\langle w^{\prime}\right\rangle$ show significant anisotropy of turbulence inside the upstream and downstream recirculation zones and LES computations have been successful in predicting the trends as well as profiles. While the mean velocities are quite low inside the vortex bubble, the root mean square (rms) fluctuations remain relatively high in comparison. This is especially true along the boundary of the vortex bubble. In combustion systems, high level of turbulence 
causes an increase in local turbulent flame speed, which dramatically effect to shorten the flame length. Figures 16 and 17 show Reynolds stresses $\left\langle u^{\prime} v^{\prime}\right\rangle$ and $\left\langle u^{\prime} w^{\prime}\right\rangle$. Given the complexity of the flow LES predicted Reynolds stresses show good agreement, particularly the calculations seems to capture the sharp changes in Reynolds stresses reasonably well.

For this case, the overall LES predictions are in reasonably good agreement with the experimental data and the vortex breakdown found to be well predicted.

\section{Flow field and characteristic features of the high-swirl case (N16S159):}

This is the highest swirl number case $\left(S_{g}=1.59\right)$ of these experiments and perceived to be the most difficult case to predict. The tangential velocity components at inlet are high in this case (see Table 1) and resultant turbulence anisotropy is considerably high.

The streamlines corresponding to the mean velocity field taken from LES predictions is shown in Figure 18. In comparison to Figures 3 and 9 the recirculation patterns are now completely different. The high swirl causes the flow to spread radially outwards creating a long stagnation zone, which extends down the centreline up to about $x=90 \mathrm{~mm}$ from the burner exist plane. In this recirculation zone three different vortices are formed at the axial distance of $x=20,30$ and $80 \mathrm{~mm}$ corresponding to radial distances of $r=10,20 \mathrm{~mm}$. By comparing Figures 9 and 18, it is interesting to note how the swirl velocity change results in a contrasting difference in flow behaviour. This has also been observed experimentally, and the LES model predictions found to correctly reproduced the flow features as described below.

The axial spread of the recirculation zone, in this high swirl number case $\operatorname{N16S159}\left(S_{g}=1.59\right)$, is much longer than that found in the other two cases. The size of the two vortices formed near to the bluff body is different as well. Two counter rotating vertices can be seen and swirling velocity of the primary annulus play a major role in generating these vortices above the bluff body. However, this case (N16S159) shows that using high swirl number alone is insufficient for the criterion of the downstream recirculation pattern, which appeared in the medium swirl case. Unlike N29S054 case, the N16S159 case has no downstream recirculation or vortex breakdown despite having a large swirl number. The prediction shows the occurrence of small vortex further downstream at about $x=80 \mathrm{~mm}$, which has been observed in the experiments (Al-Abdeli and Masri 2003). The central jet decays faster than other two cases (non-swirl and medium swirl) and the values of the 
centreline velocity is almost zero at $x=80 \mathrm{~mm}$. It is worth noting here that N29S054, which showed a downstream recirculation of some size, had the highest value of primary annulus axial velocity compared to the highest swirl number case (N16S159) which does not show a downstream re-circulation zone.

Figure 19 shows the mean axial velocity profiles at different axial locations. Although the centre line value is over-predicted at $x / D=0.6$ in general the comparison for the mean axial velocity is very good at all downstream axial locations. In this case, the mean axial velocity along the centreline does not reach negative values. In comparison to the other two cases, the decrease in axial velocity in the inner part (radial direction) results in an increase in axial velocity in the outer part of the flow. At $x / D \in\{1.4,2.0,2.5\}$ axial velocity component is much smaller than the upstream values. This occurs as a result of the high spreading rate of momentum which is an important property in swirling flows. In comparison to other cases experimental data show that decrease in axial momentum and spreading is more prominent in this case. LES has correctly reproduced this behaviour.

The comparison of the mean radial velocity is shown in Figure 20. Radial velocity which is small in magnitude shows some notable under predictions at $x / D=0.6$ and $x / D=0.8$. Figure 21 shows the comparisons for mean swirling velocity. Calculations shows the correct trend but do not the reproduce the peak values present in the outer shear layer at $x / D \in\{0.2,0.4\}$. The radial spread of the swirling velocity is slightly over-predicted in the region $r / R=0.5-1.0$ at the axial locations $x / d \in\{0.6,0.8\}$. The overall agreement appears to be reasonable for the mean swirling velocity for this highest swirl number, complex flow field.

Figure 22 shows the comparison of axial velocity fluctuations (rms). The predictions are in good agreement with experimental measurements. Shown in Figure 23 are comparisons for radial velocity fluctuations. The radial velocity fluctuation are slightly over predicted at $x / D=0.2(r / R=1.2)$ and under predicted at $x / D=0.6(r / R=0.5)$. These discrepancies may be linked to under-predictions of mean radial velocity seen in Figure 20. The overall comparison for the rms radial velocity is better than mean radial velocity comparisons. Figure 24, shows the comparison of the swirling velocity fluctuations. Minor under predictions can be seen at the last three axial locations, $x / D \in\{1.4,2.0,2.5\}$. However, results from the LES model found to capture the peaks and the trend reasonably well in the near burner region. 
Figures 25 and 26 show the comparisons for Reynolds stresses $\left\langle u^{\prime} v^{\prime}\right\rangle$ and $\left\langle u^{\prime} w^{\prime}\right\rangle$. LES calculations show highly varying profiles of Reynolds stresses along the radial direction for both stresses. Near burner predictions are reasonably good except at the centre line. Further down stream experimental data do not show the degree of variation seen in the predictions. However, overall magnitudes of the predicted Reynolds stresses agree with the experimental values. The variations in predictions could be due to the high swirl number and the complexity of the turbulence flow field. Measurement of $\left\langle u^{\prime} w^{\prime}\right\rangle$ stress also shows considerable degree of highly varying nature up to $x / D=0.8$. Reynolds stresses are harder to compute accurately and depends on predictive successes of other velocity components. Since this is a highly swirling case, the flow field consists of a number of high shear regions. Given the complexity of the flow it is encouraging to find that the LES model produces reasonably good predictions for the Reynolds stresses..

In all three cases, considered in this study, it can be concluded that the LES modelling technique is able to predict all important flow features reasonably well. In particular, the model, has successfully predicted the mean velocity components, their fluctuations and the flow features such as bubble type vortex break down as observed in the experiments. With this success in predicting isothermal cases, considered in this study, it is the authors intention to extend this work to reacting cases in this burner configuration where data is available for species concentrations, temperature and other important parameters such as mixture fraction and mixture fraction variance. That will be the next phase of this work.

\section{CONCLUSIONS}

In this paper, Large Eddy Simulations have been carried out for isothermal swirling flows based on the Sydney swirl burner experimentally investigated by Al-Abdeli and Masri (2003). A set of detailed experimental data including mean velocities, rms fluctuations and Reynolds stress components are available for validation. Three different test cases have been modelled under various flow conditions. The simulations show that with appropriate inflow, outflow boundary conditions and sufficient grid resolution LES successfully simulated the experimentally observed structure and topology of the swirling flow fields and agree well with measured mean velocity, rms fluctuations and the Reynolds shear stresses.

In the non-swirling case turbulence is mostly generated in the shear layer near sudden expansion at the bluff body wall. In the swirling flows turbulence is not only generated in the shear layer near the sudden expansion, but also in the vortex breakdown (VB) bubble. It is 
found that the centre-line axial flow recirculates or break down (bubble type vortex breakdown) is achieved in the flow with a moderate swirl number (N29S054), rather than the high swirl number (N16S159). However, it is evident from the results that the axial momentum (or Reynolds number) of the swirling annulus plays a major role in determining the onset of vortex breakdown. The combination of low swirl number and higher primary axial velocity seen to cause the vortex breakdown. Experimentally observed features of vortex breakdown and flow structures at different operating conditions were successfully reproduced by the present large eddy simulations indicating that LES is capable of predicting VB phenomena which occurs only at certain conditions.

The simulations have been able to capture the fast decay of turbulence in swirling flows due to the fast transport of turbulent kinetic energy towards radial direction as a result of the high centrifugal force induced by swirling motion and this helps to reproduce the important recirculation zones seen in experimental results. The results of this study show that LES seems to be more suitable for such complex flows. To fully appreciate the present effort, the results presented here have been compared with those presented at the Turbulent NonPremixed Flames (TNF8) workshop (2006) and the results presented in this paper were seen to be very good compared with results obtained by other international groups working in this series of flames.

While, a considerable amount of work has been carried out here (dynamics of isothermal swirling flow field), some efforts are still required to improve the accuracy, applicability and efficiency of the LES technique to the field of turbulent combustion. The computational effort required to accomplish the present simulations was reasonably moderate, confirming that LES has a great potential in engineering applications. With further advancement of computer hardware and software technologies LES could be more useful for general engineering flow applications.

\section{REFERENCES}

Al-Abdeli, Y.M, Masri, A.R. (2003), Recirculation and flow field regimes of unconfined nonreacting swirling flow, Exp. Therm. Fluid Sci., Vol. 23, pp. 655-665.

Al-Abdeli, Y.M. (2003), Experiments in Turbulent swirling non-premixed flame and isothermal flows, PhD Thesis, School of Aero. Mech. Mecha. Eng., University of Sydney, Australia. 
Ahmed, S.A. (1998), Velocity measurements and turbulence statistics of a confined isothermal swirling flow, Exp. Therm. Fluid Sci., Vol. 17, pp. 256-270.

Bell, J.B. Colella, P. and Glaz, H.M. (1989), A second order projection method for the incompressible Navier-Stokes equations, J. Comp. Phys., Vol. 85, pp. 257-283.

Billent, P, Chomaz, J.M, Huerre, P. (1998), Experimental study of vortex breakdown in swirling jets, J. Fluid Mech., Vol.376, pp.183-210.

Branley, N and Jones,W.P. (2001), Large eddy simulation of a turbulent non-premixed flame, Combust. Flame, Vol.127, pp.1914-1934.

Branley, N. (1999), Large eddy simulation of Turbulent non-premixed flames, PhD Thesis, Dep. Chem. Eng., Imperial college of Science and Technology, UK.

Bear, J.M. and Chigier, N.A. (1972), Combustion Aerodynamics, Wiley, New-York, NY.

Carati, D., Ghosal, S and Moin, P. (1995), On the representation of backscatter in dynamic localization models, Phys. Fluids, Vol.7, pp.606-616.

Dally, B, Masri, A. R. Barlow, R. S and Feitchner, G. J. (1998), Instantaneous and mean compositional structure of bluff body stabilized non-premixed flames, Combust. Flame, Vol. 114, 119-148.

Deardoff, J,W. (1970), A numerical study of three dimensional turbulent channel flow at large Reynolds numbers, J. Fluid Mech., Vol.41, pp.453-480.

Dellenback, P. A, Metzger, D. E, Neitzel, G. (1988), Measurements in turbulent swirling flow through an abrupt axi-symmetric expansion, AIAA Journal, Vol. 26(6), pp. 669-681.

Di Mare, Jones. W. Menzies. K. (2004), Large eddy simulation of a model gas turbine combustor, Combust. Flame, Vol.137(3), 278-294.

Escudier, M. (1988). Vortex breakdown: observations and explanations, Prog. Aero. Sci., Vol. 25, pp. 189-229.

Escudier, M., Zehinder, N. (1982). Vortex-flow regimes, J. Fluid Mech., Vol. 115, pp. 105-121.

Germano, M. ,Piomelli, U., Moin, P. and Cabot, W.H. (1991), Dynamic subgrid eddy viscosity model, Phy. Fluids, Vol. 3, pp. 1760-1765.

Ghosal, S, Lund, T.S and Moin, P. (1992), A dynamic localization model for large eddy simulation of turbulent flows, Center for Turbulence Research, Stanford University, USA.

Gresho, P. (1990), On the theory of semi-implicit projection methods for viscous incompressible flow and its implementation via finite element method that also introduces a nearly consistent mass matrix, Int. J. Numerical methods and Fluids, Part I, pp.587-620. 
Guo, B, Langrish, T.A.G, Fletcher, D.F. (2001), Simulation of turbulent swirl flow in an axisymmetric sudden expansion, AIAA J., Vol.39, pp.96-102.

Gupta, A.K., Beer, J.M. and Swithenbank, J. (1977), Concentric multi-annular swirl burner: stability limits and emission characteristics, Sixteenth Symposium (Int.) on Combustion, The Combustion Institute, pp. 79-91.

Kim, W, Menon. S, Mongia, H. (1999), Large eddy simulation of a gas turbine combustor flow, Combust. Sci. Tech., 143, 25-63.

Kitoh, O. (1991), Experimental study of turbulent swirling flow in a straight pipe, J. Fluid Mech., Vol.225, pp.445-465.

Kirkpatrick, M.P. (2002), Large eddy simulation code for industrial and environmental flows, PhD Thesis, Sch. Aero. Mech. and Mecha. Eng, University of Sydney, Australia.

Kirkpatrick, M.P, Armfield, S.W, Kent, J.H. (2003a), A representation of curved boundaries for the solutions of the Navier-Stokes equations on a staggered three dimensional Cartesian grid, J. Comput. Phy.,Vol 104, pp.1-36.

Kirkpatrick, M.P, Armfield, S.W, Masri, A.R and Ibrahim, S.S. (2003b), Large eddy simulation of a propagating premixed flame, Flow Turb. Combust., Vol 70, pp. 1-19.

Kirkpatrick, M.P, Mansour, N.N., Ackerman, A.S. and Stevens, D.E. (2003c), Dynamics turbulence modelling in large-eddy simulation of the cloud-topped atmospheric boundary layer, Annual research brief, Centre for Turbulence Research, Stanford University.

Kempf, A, Lindstedt, R.P, Janika, J. (2006), Large eddy simulation of bluff body stabilized nonpremixd flame, Combust. Flame., Vol. 144, pp.170-189.

Lucca-Negro, O. and O’Doherty, T.O. (2001), Vortex breakdown: a review, Prog. Ener. Comb. Sci., Vol. 27, pp. 431-481.

Masri, A.R., Pope, S.B. and Dally, B.B. (2000), Probability density function computation of a strongly swirling nonpremixed flame stabilised on a new burner, Proc. Combut. Inst., Vol. 28, pp. 123-131.

Masri, A.R., Kalt, P.A.M., Barlow, R.S. (2004). The compositional structure of swirlstabilised turbulent nonpremixed flames, Combut. Flame, Vol. 137, pp. 1-37.

Mahesh, K, Constantinescu, G, Apte, S, Iaccarineo, G, Ham, F, Moin, P. (2005), Large eddy simulation of reacting turbulent flows in complex geometries, ASME J. App. Mech., Vol.73, pp.374-381.

Nathan, G.J, Turns, Hill, S.J and Luxton, R.E. (1998), An axisymmetric fluidic nozzle to generate jet precession, J. Fluid Mech., Vol. 370, pp.347-380.

Novak, F. and Sarpkaya, T. (2000). Turbulent Vortex breakdown at high Reynolds numbers, AIAA J., Vol. 38, pp. 825-834. 
Pierce, C.D. and Moin, P. (1998), Method for generating equilibrium swirling inflow conditions, AIAA J., Vol. 36, pp. 1325-1327.

Piomelli, U. and Liu, J. (1995), Large eddy simulation of channel flows using a localized dynamic model, Phy. Fluids, Vol. 7, pp. 839-848.

Proceedings of the $8^{\text {th }}$ International workshop on Turbulent Non-premixed Flames. (2006), Heidelberg, Germany, pp.11-75.

Sarpkaya, T. (1971). On stationary and traveling vortex breakdowns, J. Fluid Mech., Vol. 45, pp. 545-559.

Sankaran, V, Menon, S. (2002), LES of spray combustion in swirling flows, J. Turbulence, Vol.3, pp.11-23.

Smagorinsky, J. (1963), General circulation experiments with the primitive equations”, M. Weather Review, Vol.91, pp.99-164

Sloan, D.G., Smith, P.J. and Smoot, L.D. (1986). Modelling of Swirl in Turbulent Flow Systems, Prog. Energy Combust Sci., Vol. 12, pp. 163-250.

Syred, N and Beer, J.M. (1974), Combustion in Swirling Flows: A Review, Combust. Flame, 23, pp. 143-201.

TNF8 Workshop (2006), http://www.ca.sandia.gov/TNF/8thWorkshop/index.php.

Van Kan, J. (1986). A second order accurate pressure correction scheme for viscous incompressible flow, SIAM J Sci. Stat Comput., Vol. 7, pp. 870-891.

Venkatramanan, R, Pitsch, H. (2005), Large eddy simulation of bluff body stabilized nonpremixed flame using a recursive filter refinement procedure, Combust. Flame, Vol.142, pp.329-347.

Weber, R, Visser, B.M, Boysan, F. (1990), Assessment of turbulent modelling for engineering prediction of swirling vortices in the near burner zone, Int. J. Heat and Fluid Flow, Vol.11, pp.225-238.

Wall, C.T and Moin, P. (2005), Numerical methods for Large eddy simulation of acoustic combustion instabilities, Tech. Rep. TF.91. Stanford University, CA.

Wang.P and Bai, X,S, Wessman, M and Klingmann, J. (2004), Large eddy simulation and experimental studies of a confined turbulent swirling flow, Phy. Fluids, Vol.16, pp.33063324. 


\section{FIGURE CAPTIONS}

Figure 1: Schematic drawing of the Sydney swirl burner (adapted from Al-Abdeli and Masri, 2003).

Figure 2: LES predicted time averaged mean and rms fluctuations at $x / D=0.4$ using different grid resolutions for case N16S159. Dotted lines represent the Grid 1 results (coarser grid), dashed lines represent the Grid 2 results (finer grid), and symbols represent experimental measurements.

Figure 3: Streamlines generated from the mean velocity field of the non-swirl case (N21S00) obtained from LES calculation.

Figure 4: Comparison of mean axial velocity. Lines represent LES results, and symbols represent experimental measurements.

Figure 5: Comparison of mean radial velocity. Lines represent LES results, and symbols represent experimental measurements.

Figure 6: Comparison of rms of axial velocity fluctuations. Lines represent LES results, and symbols represent experimental measurements.

Figure 7: Comparison of rms of radial velocity fluctuations. Lines represent LES results, and symbols represent experimental measurements.

Figure 8: Comparison of Reynolds shear stress $\left\langle u^{\prime} v^{\prime}\right\rangle$. Lines represent LES results, and symbols represent experimental measurements.

Figure 9: Streamlines generated from the mean velocity field of the moderate swirl case (N29S054) obtained from LES calculation

Figure 10: Comparison of mean axial velocity. Lines represent LES results, and symbols represent experimental measurements.

Figure 11: Comparison of mean radial velocity. Lines represent LES results, and symbols represent experimental measurements.

Figure 12: Comparison of mean swirl velocity. Lines represent LES results, and symbols represent experimental measurements.

Figure 13: Comparison of rms of axial velocity fluctuations. Lines represent LES results, and symbols represent experimental measurements.

Figure 14: Comparison of rms of radial velocity fluctuations. Lines represent LES results, and symbols represent experimental measurements.

Figure 15: Comparison of rms of swirl velocity fluctuations. Lines represent LES results, and symbols represent experimental measurements.

Figure 16: Comparison of Reynolds shear stress $\left\langle u^{\prime} v^{\prime}\right\rangle$. Lines represent LES results, and symbols represent experimental measurements. 
Figure 17: Comparison of Reynolds shear stress $\left\langle u^{\prime} w^{\prime}\right\rangle$. Lines represent LES results, and symbols represent experimental measurements.

Figure 18: Streamlines generated from the mean velocity field of the high swirl case (N16S159) obtained from LES calculation

Figure 19: Comparison of mean axial velocity. Lines represent LES results, and symbols represent experimental measurements.

Figure 20: Comparison of mean radial velocity. Lines represent LES results, and symbols represent experimental measurements.

Figure 21: Comparison of mean swirl velocity. Lines represent LES results, and symbols represent experimental measurements.

Figure 22: Comparison of rms of axial velocity fluctuations. Lines represent LES results, and symbols represent experimental measurements.

Figure 23: Comparison of rms of radial velocity fluctuations. Lines represent LES results, and symbols represent experimental measurements.

Figure 24: Comparison of rms of swirl velocity fluctuations. Lines represent LES results, and symbols represent experimental measurements.

Figure 25: Comparison of Reynolds shear stress $\left\langle u^{\prime} v^{\prime}\right\rangle$. Lines represent LES results, and symbols represent experimental measurements.

Figure 26: Comparison of Reynolds shear stress $\left\langle u^{\prime} w^{\prime}\right\rangle$. Lines represent LES results, and symbols represent experimental measurements. 


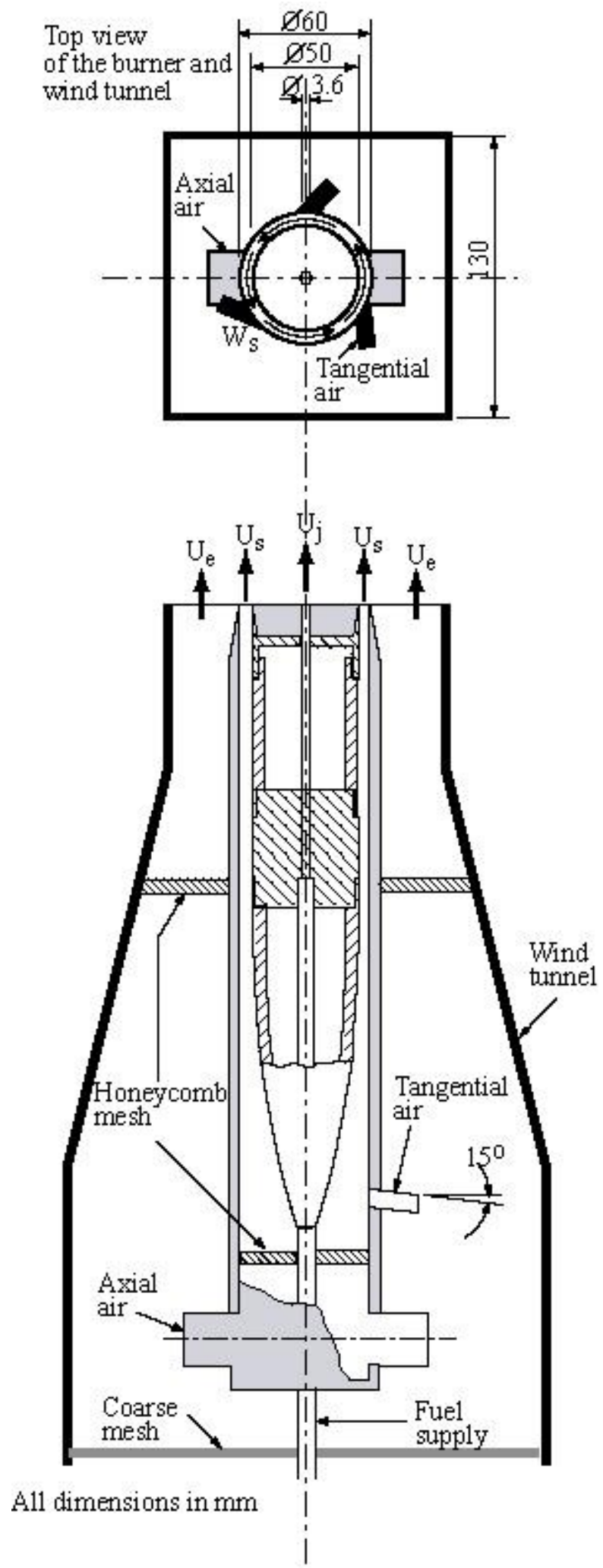

Figure 1: Schematic drawing of the Sydney swirl burner (adapted from Al-Abdeli and Masri, 2003). 

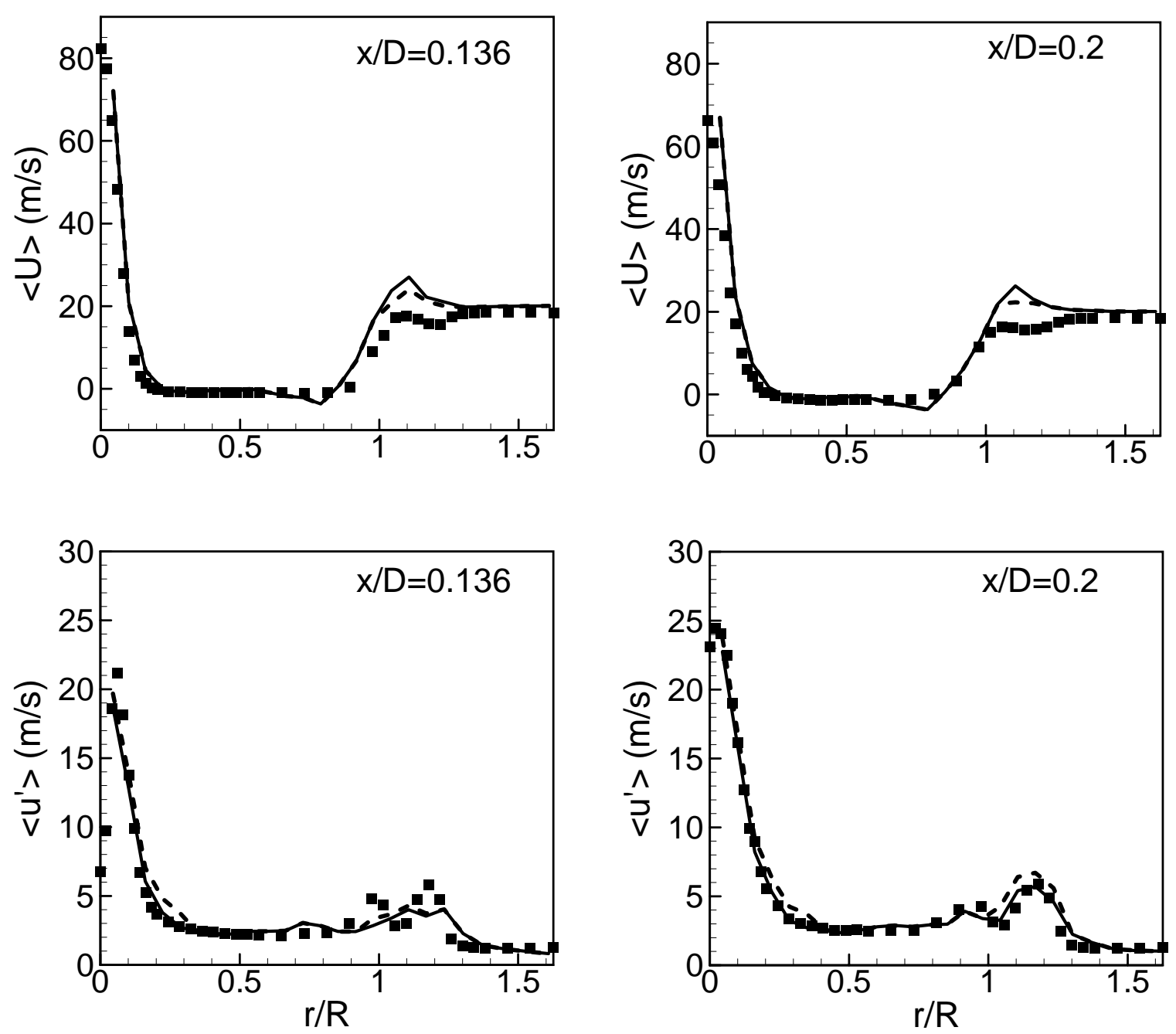

Figure 2: LES predicted time averaged mean and rms fluctuations of axial velocity at $x / D=0.136,0.2$ using different grid resolutions for case N16S159. Solid lines represent the Grid 1 results (1 million grid points), dashed lines represent the Grid 2 results (1.44 million grid points), and symbols represent experimental measurements. 


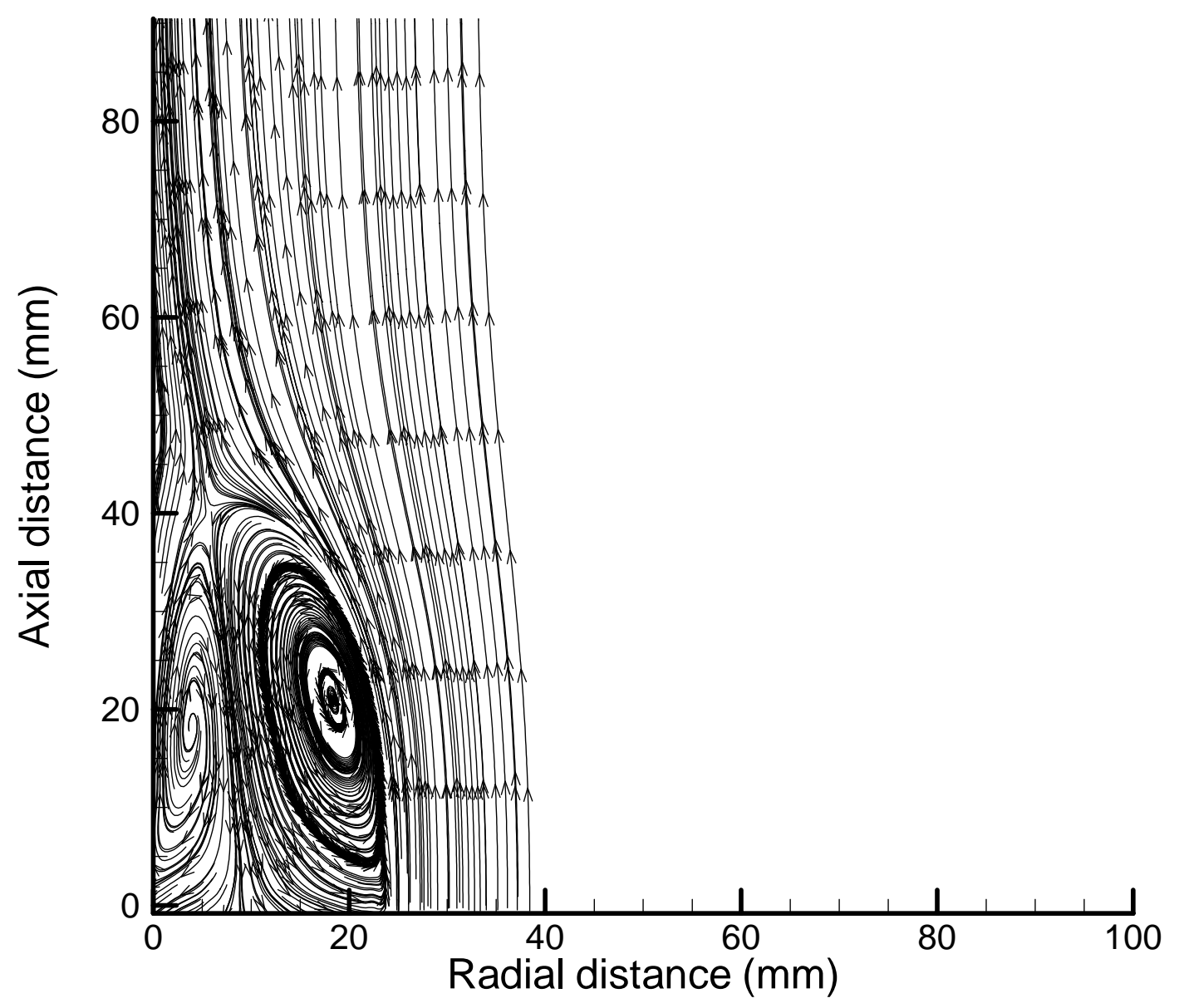

Figure 3: Streamlines generated from the mean velocity field of the non-swirl case (N21S00) obtained from LES calculation. 

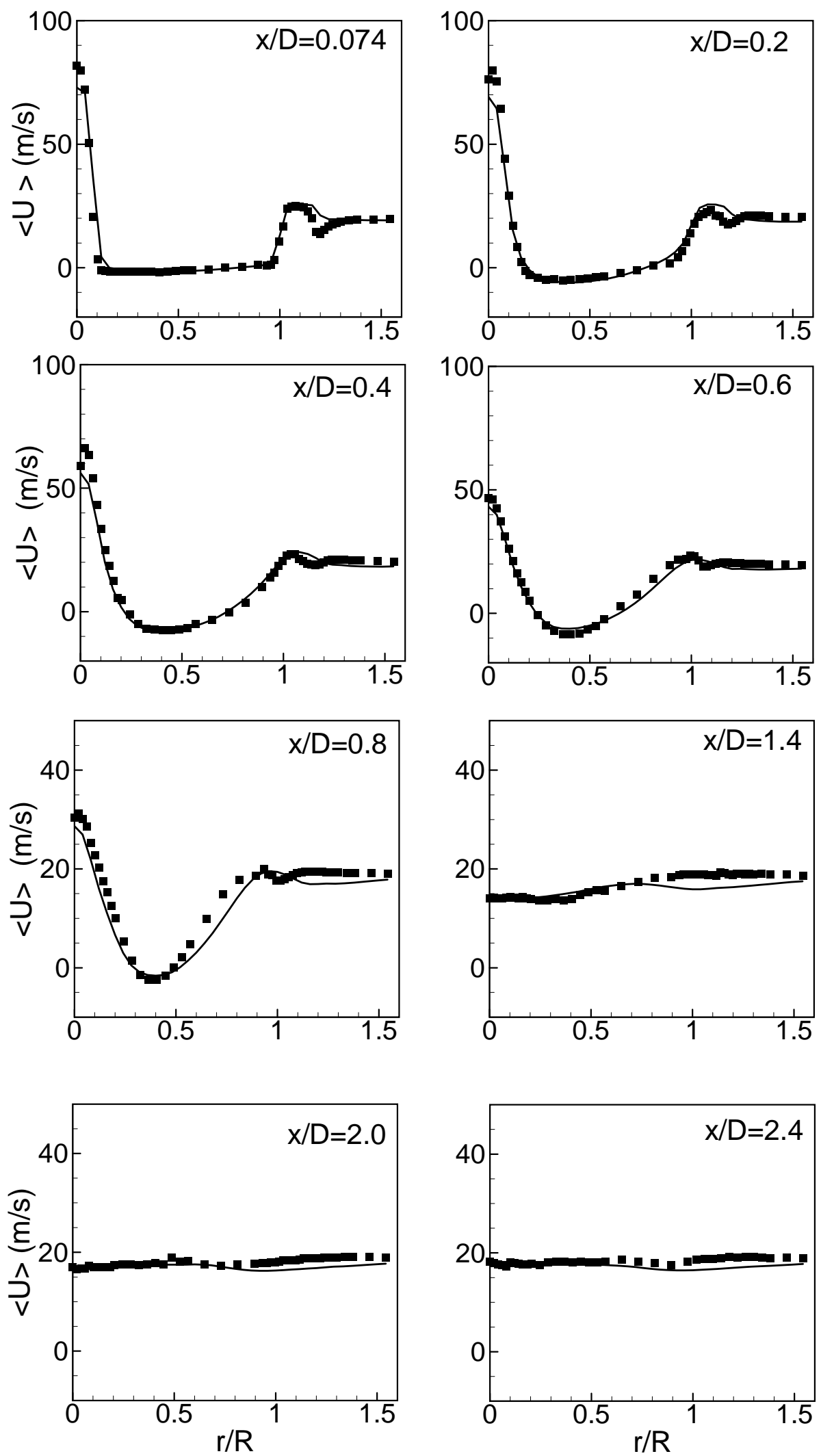

Figure 4: Comparison of mean axial velocity. Lines represent LES results, and symbols represent experimental measurements. 

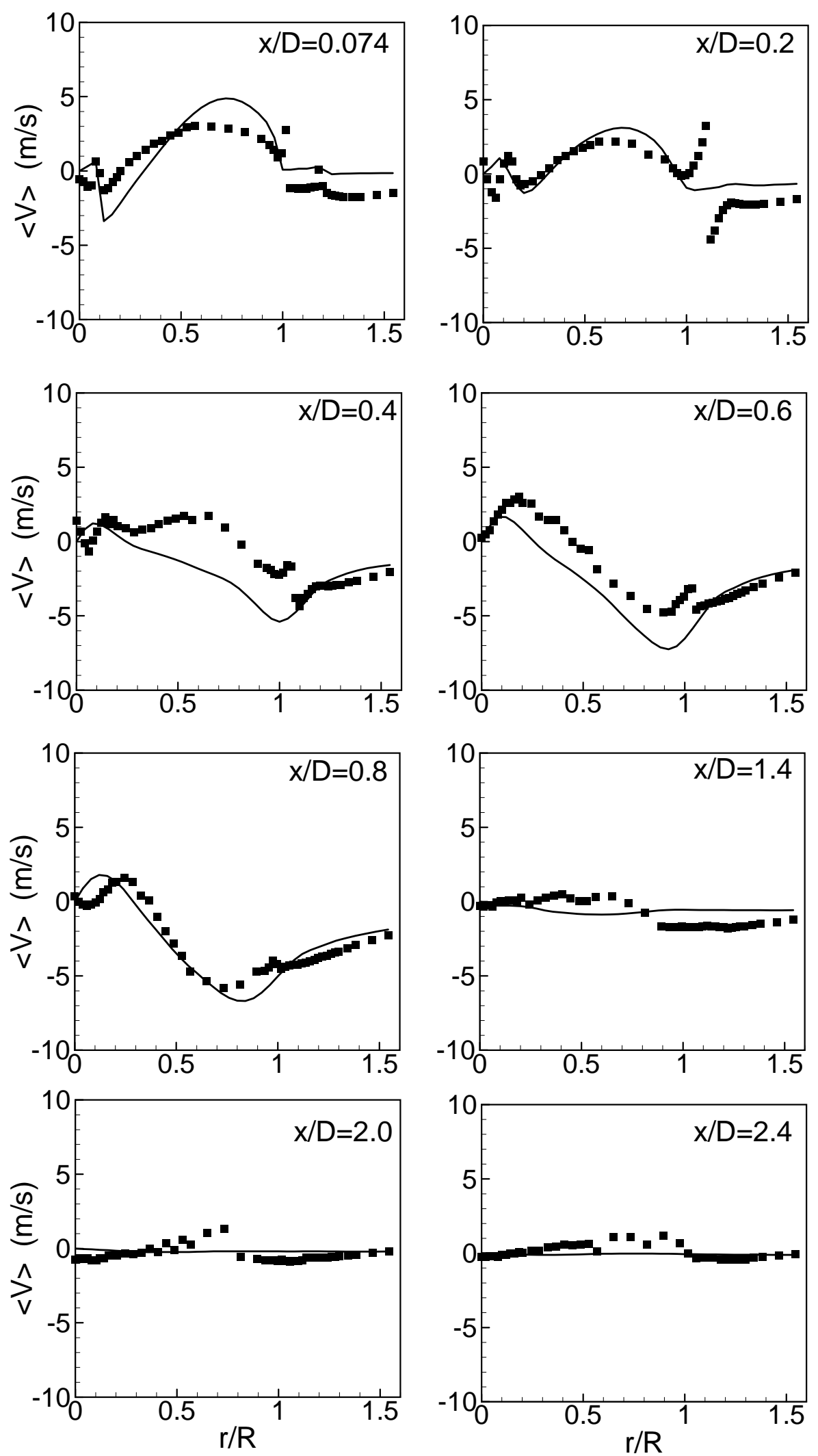

Figure 5: Comparison of mean radial velocity. Lines represent LES results, and symbols represent experimental measurements. 

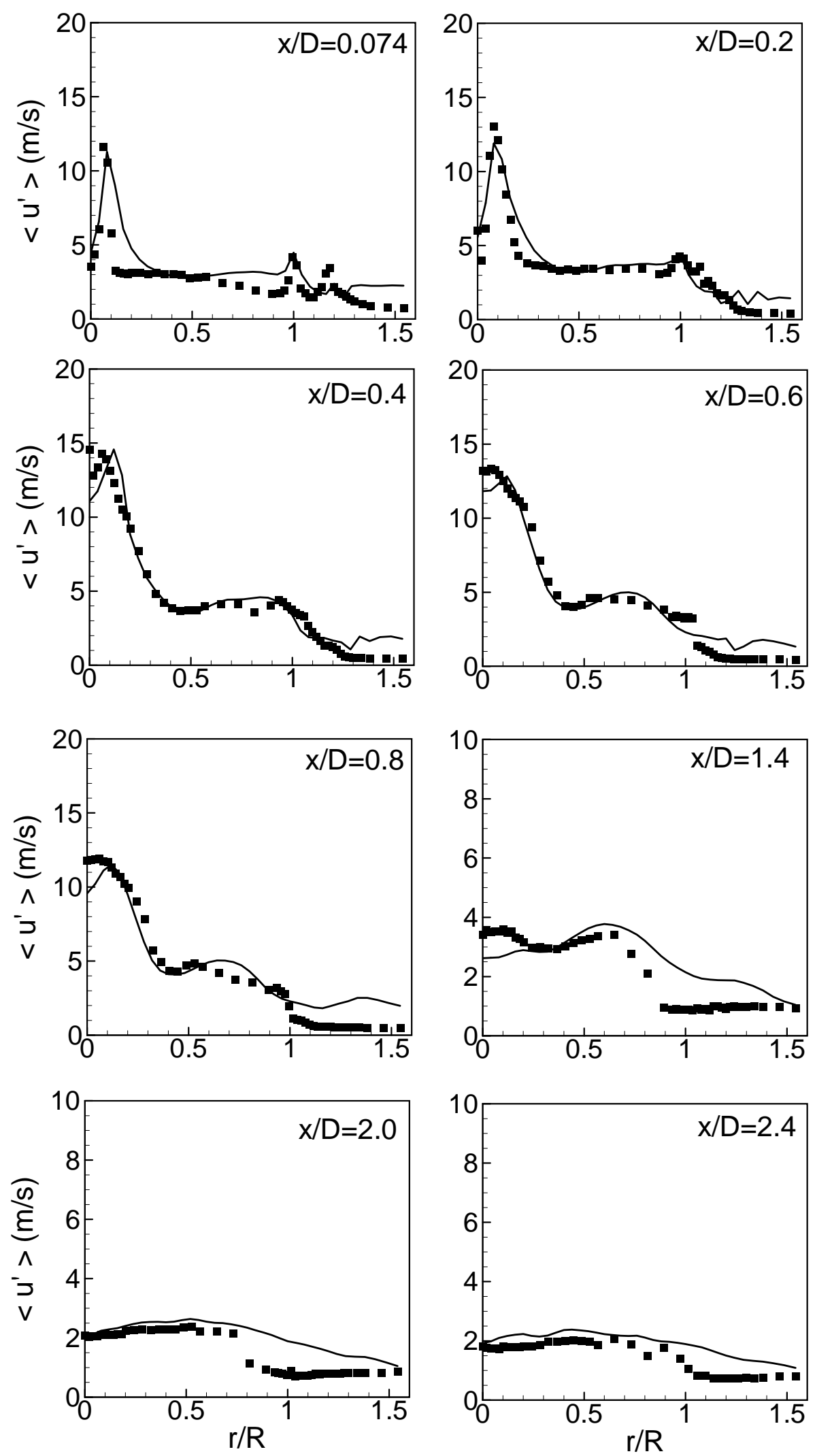

Figure 6: Comparison of rms of axial velocity fluctuations. Lines represent LES results, and symbols represent experimental measurements. 

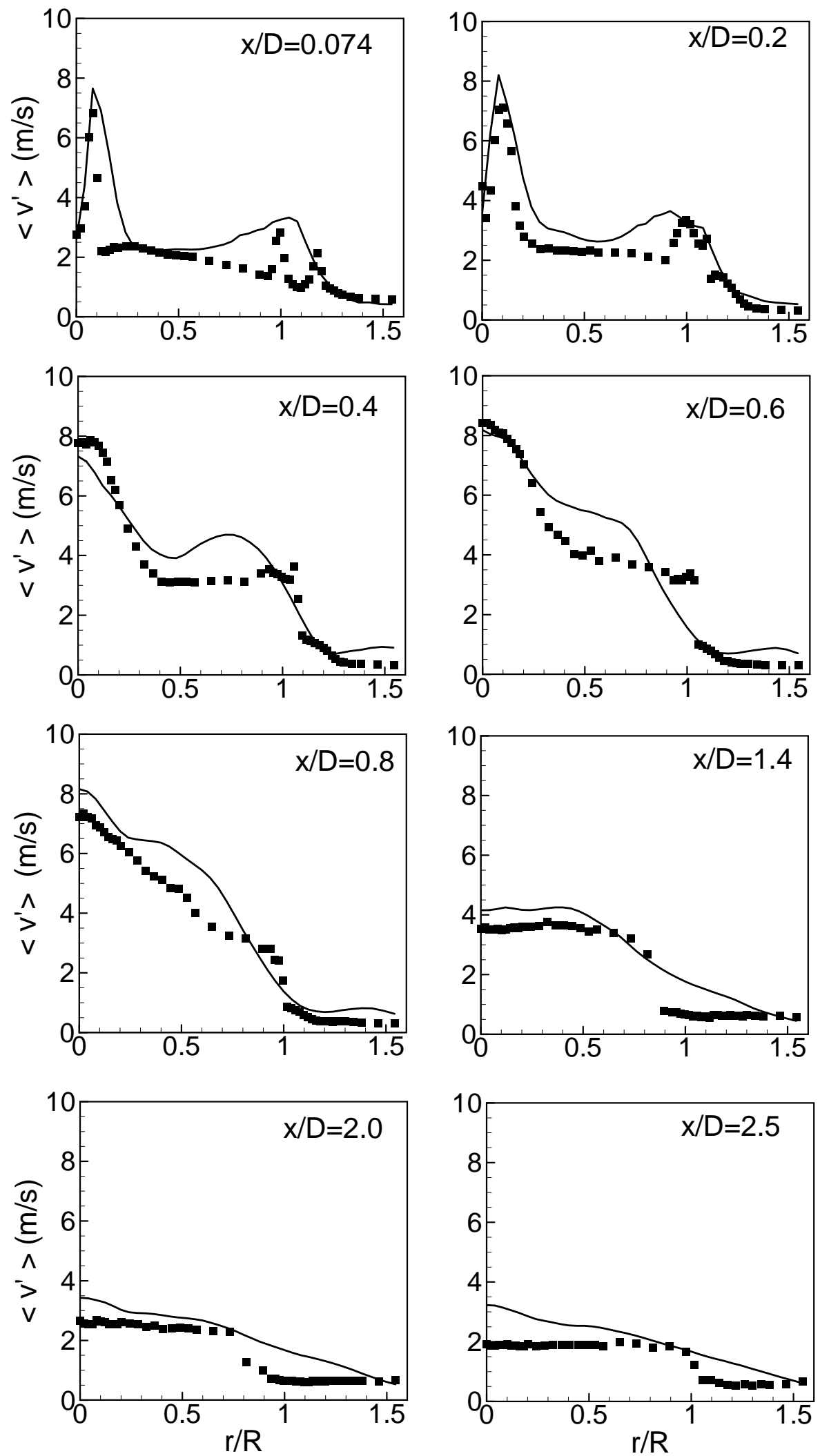

Figure 7: Comparison of rms of radial velocity fluctuations. Lines represent LES results, and symbols represent experimental measurements. 

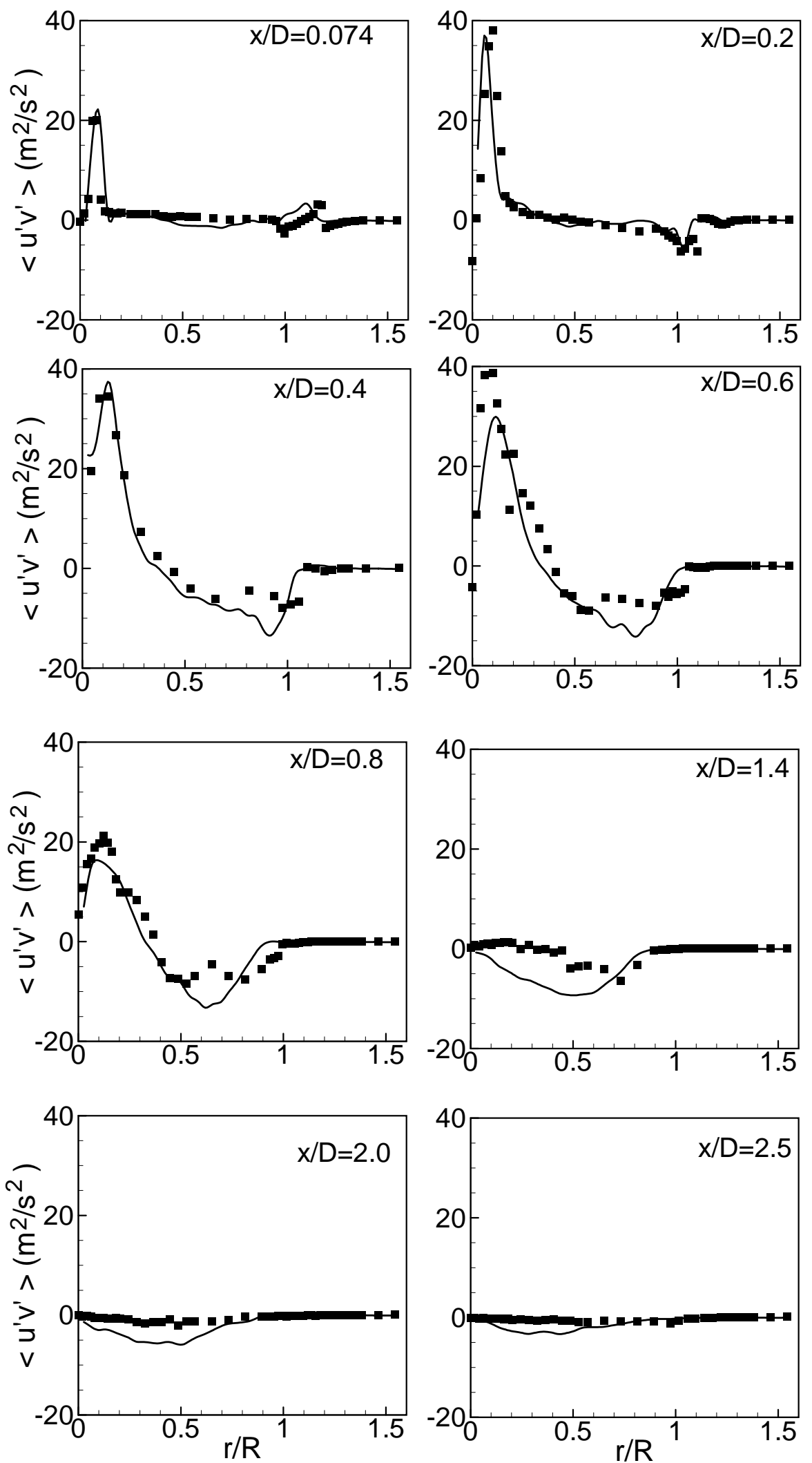

Figure 8: Comparison of Reynolds shear stress $\left\langle u^{\prime} v^{\prime}\right\rangle$. Lines represent LES results, and symbols represent experimental measurements. 


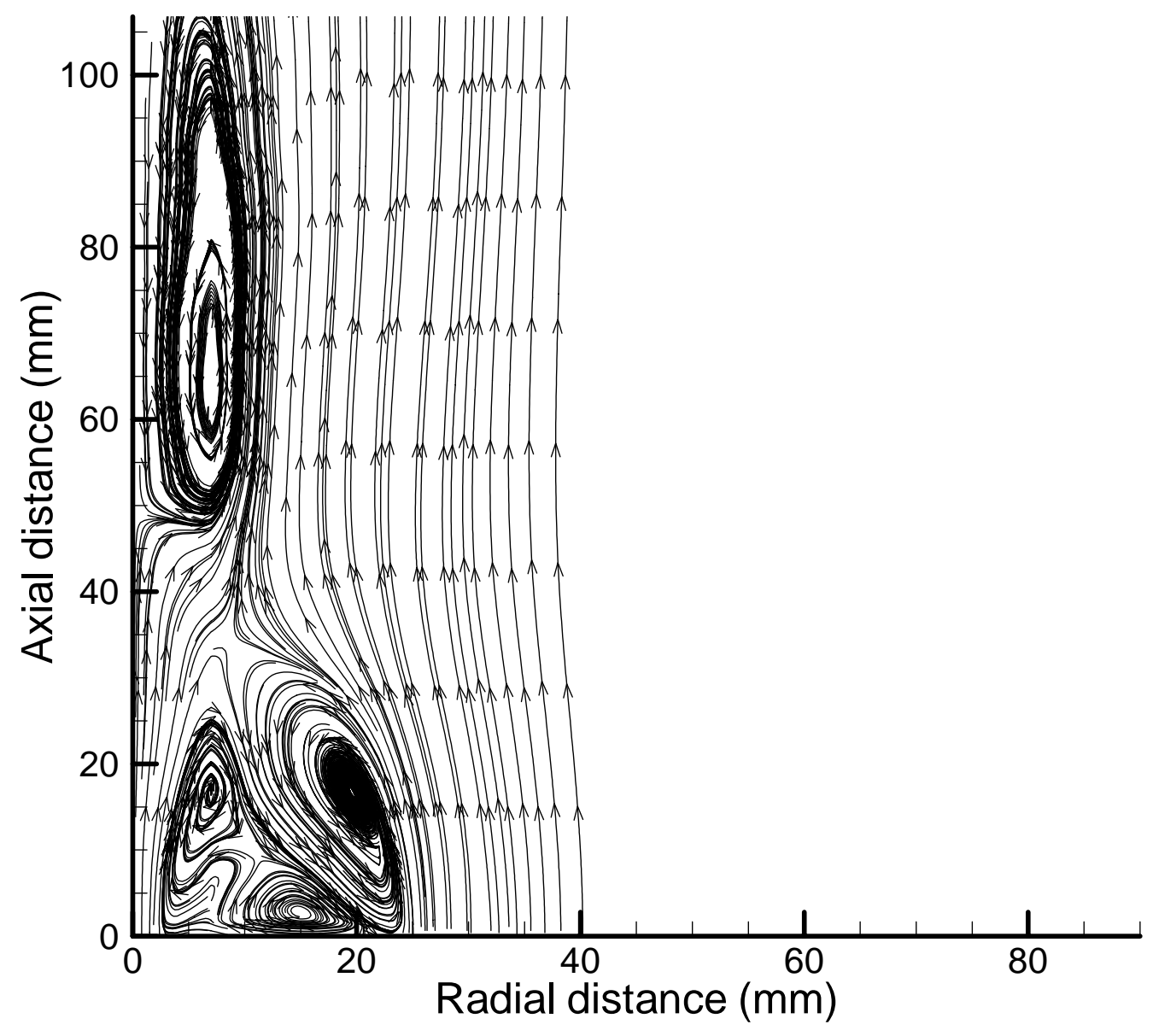

Figure 9: Streamlines generated from the mean velocity field of the moderate swirl case (N29S054) obtained from LES calculation. 

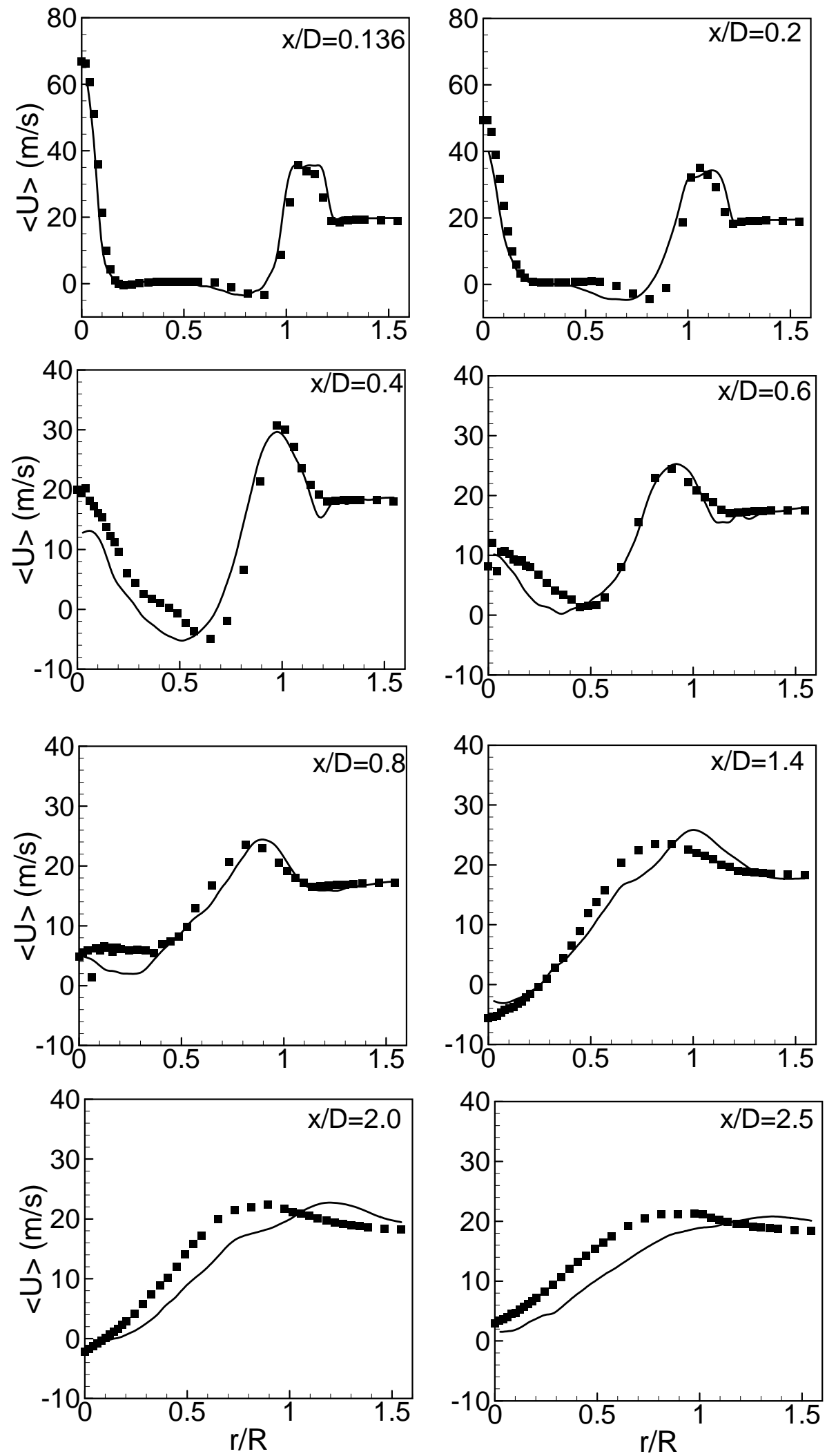

Figure 10: Comparison of mean axial velocity. Lines represent LES results, and symbols represent experimental measurements. 

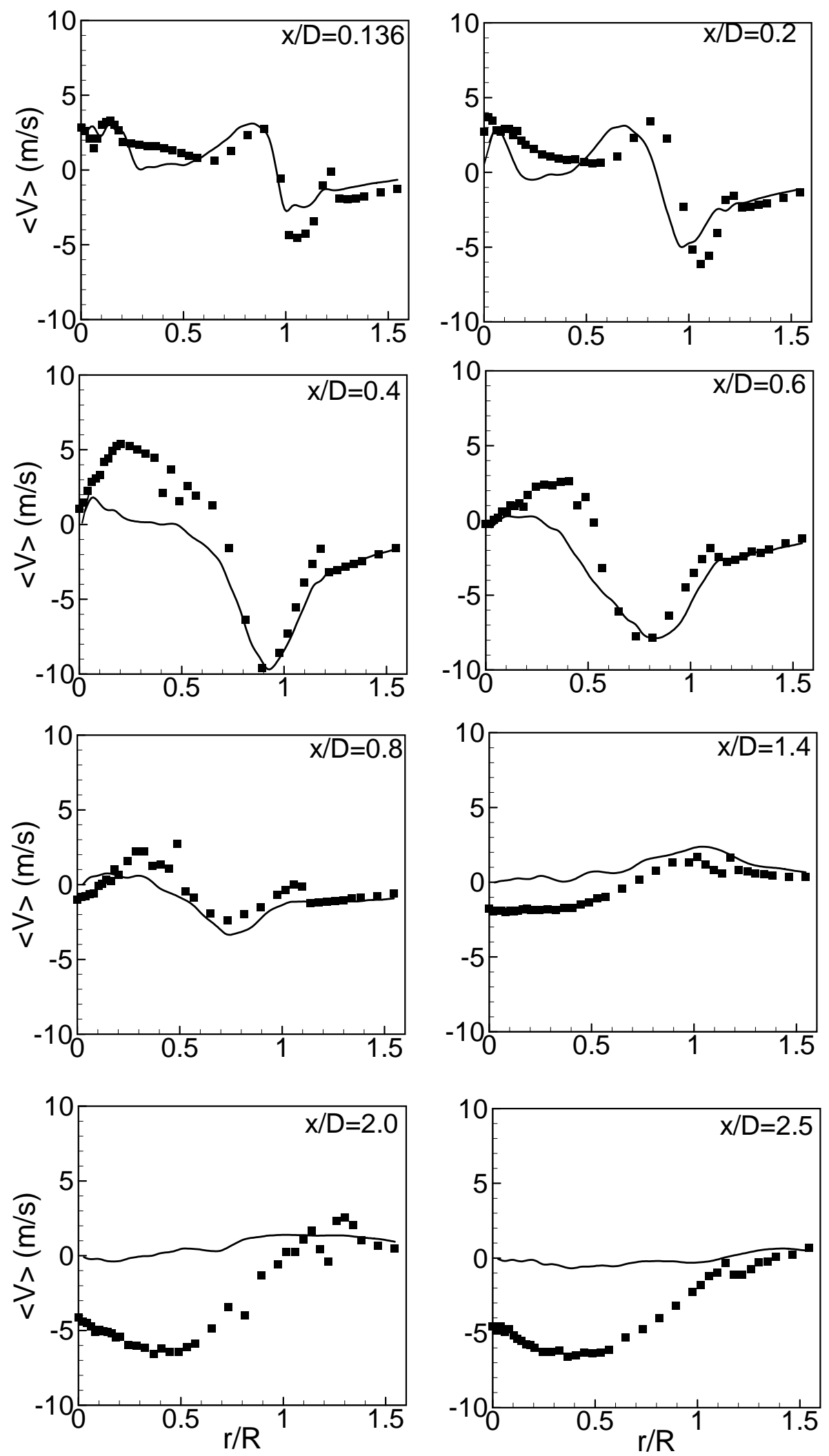

Figure 11: Comparison of mean radial velocity. Lines represent LES results, and symbols represent experimental measurements. 

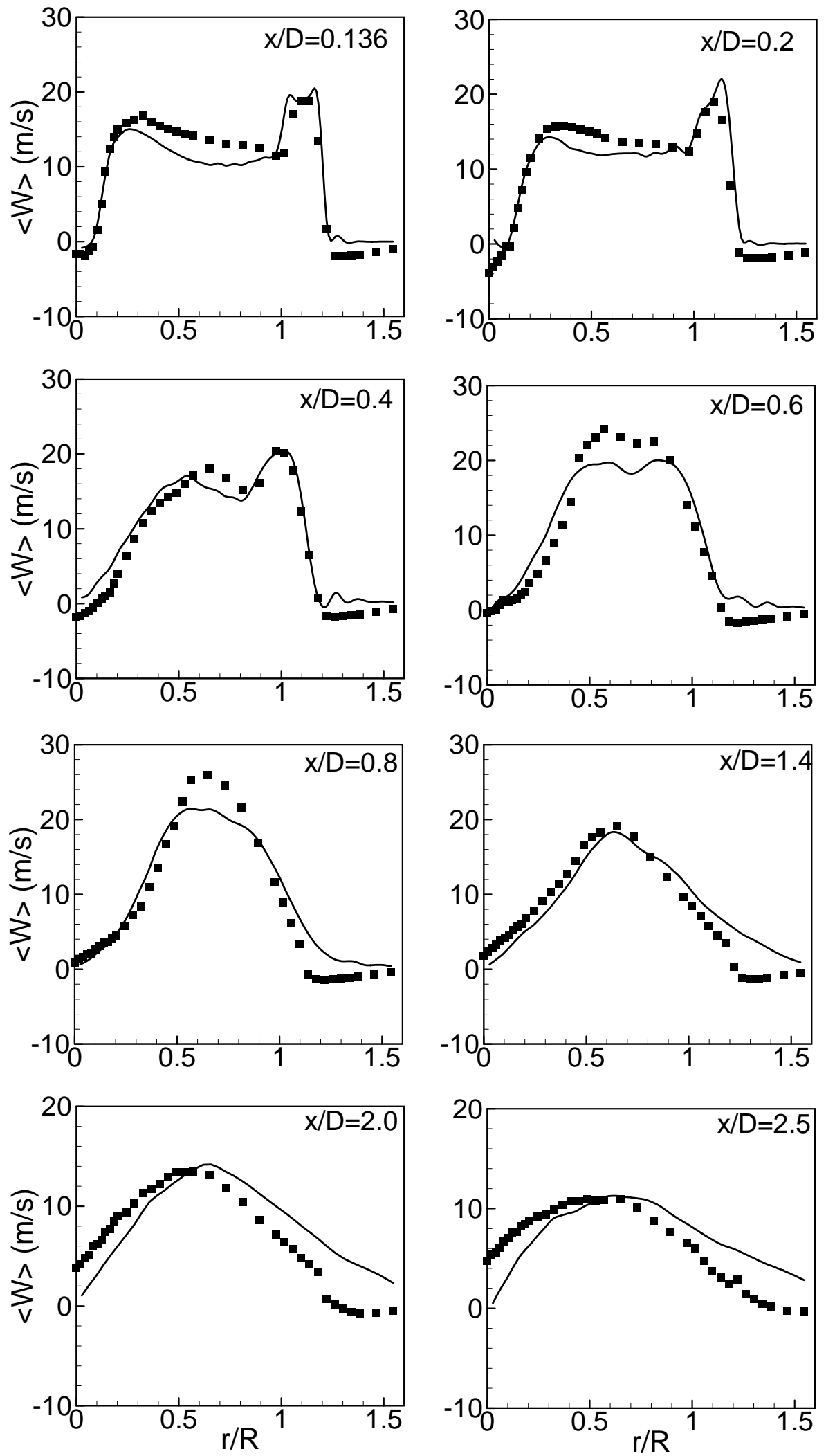

Figure 12: Comparison of mean swirl velocity. Lines represent LES results, and symbols represent experimental measurements. 

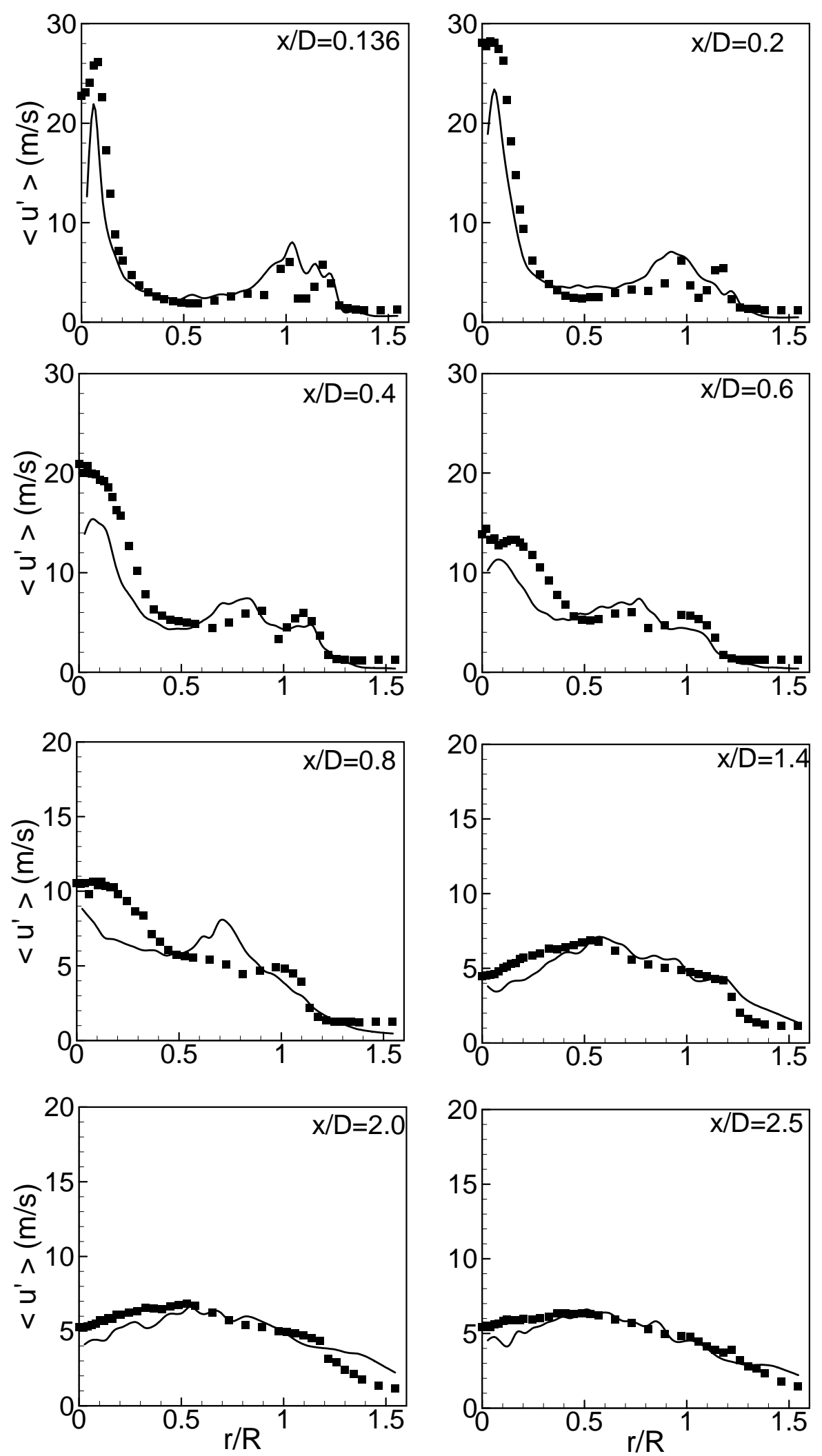

Figure 13: Comparison of rms of axial velocity fluctuations. Lines represent LES results, and symbols represent experimental measurements. 

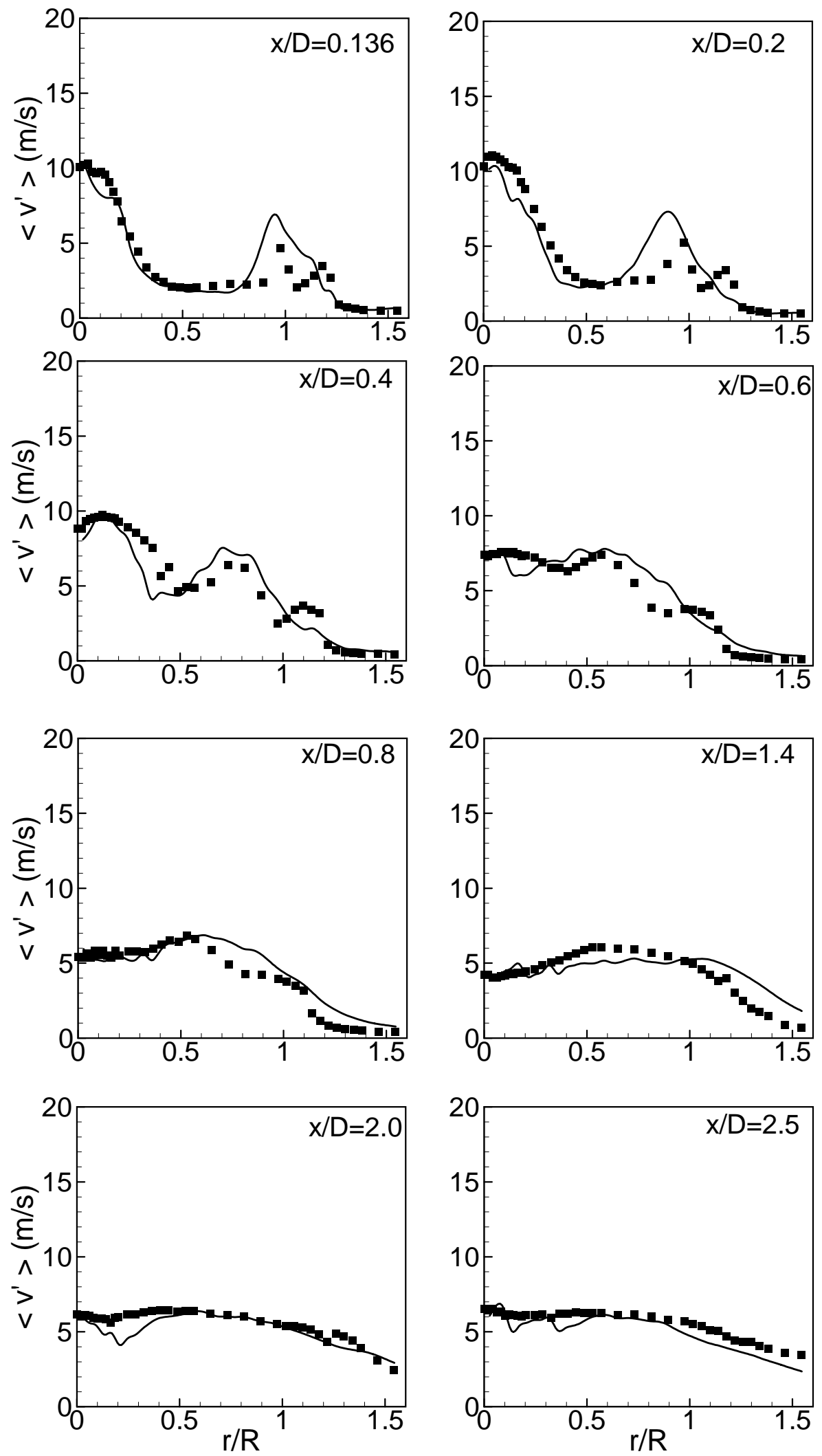

Figure 14: Comparison of rms of radial velocity fluctuations. Lines represent LES results, and symbols represent experimental measurements. 

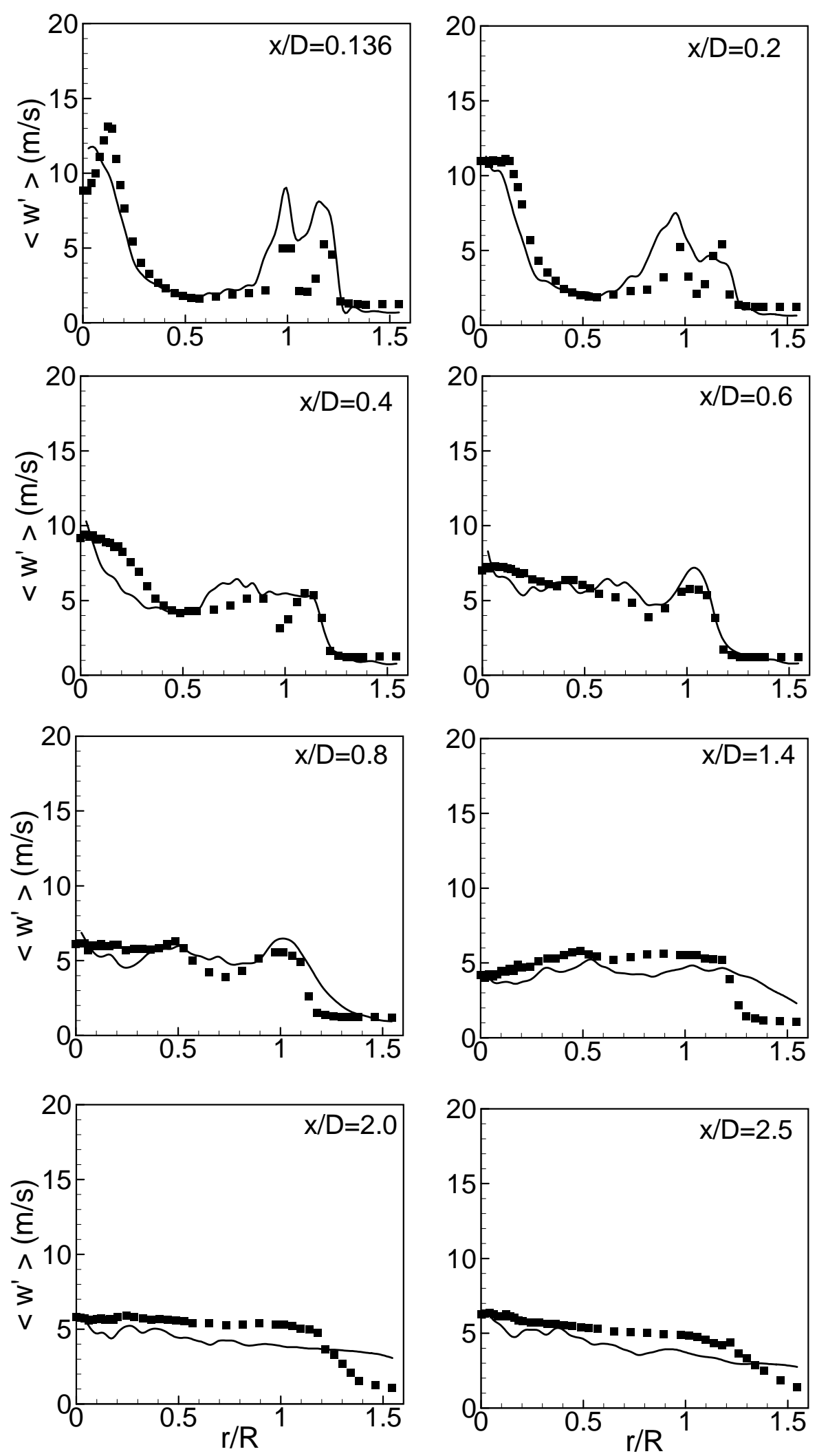

Figure 15: Comparison of rms of swirl velocity fluctuations. Lines represent LES results, and symbols represent experimental measurements. 

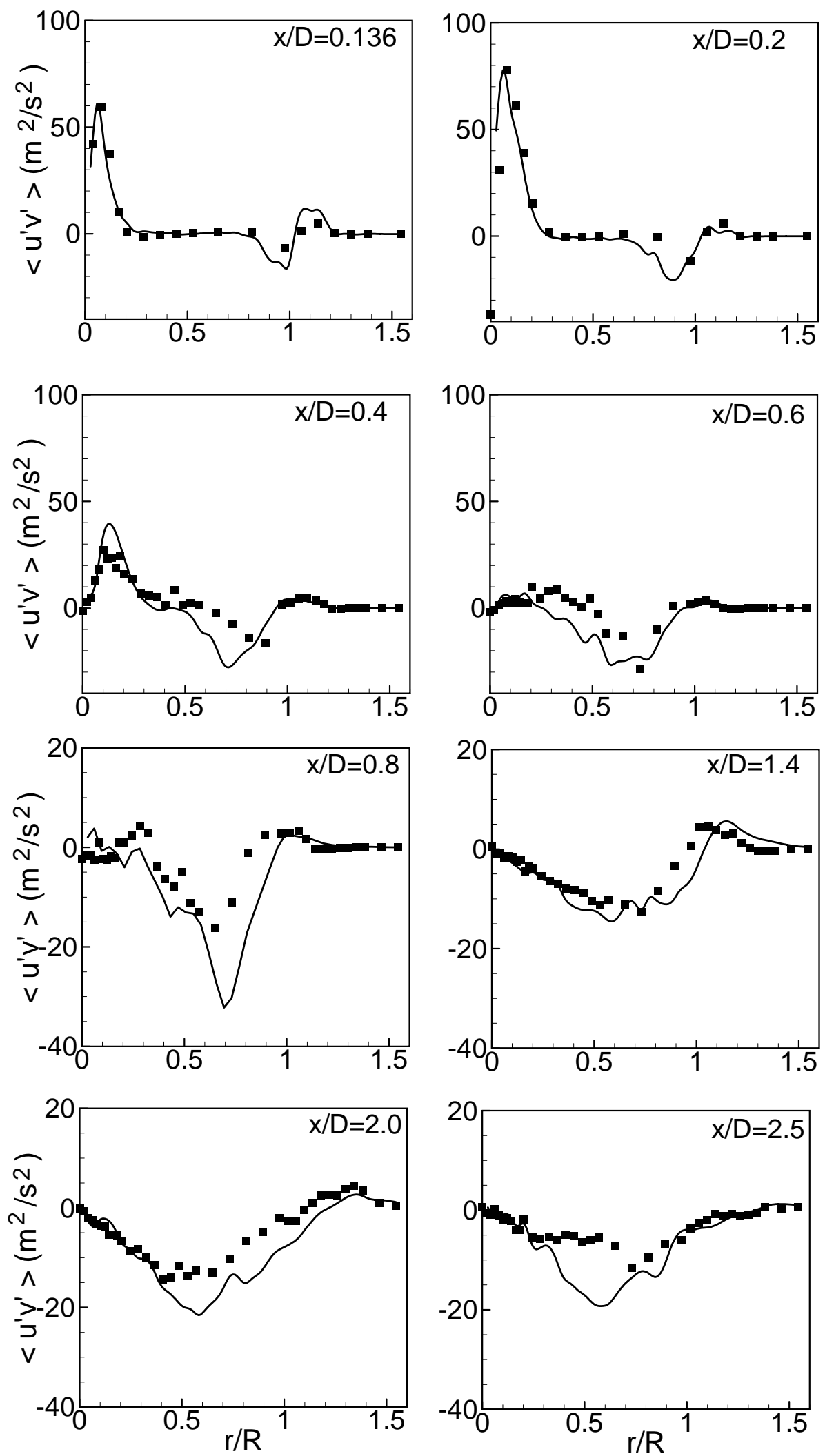

Figure 16: Comparison of Reynolds shear stress $\left\langle u^{\prime} v^{\prime}\right\rangle$. Lines represent LES results, and symbols represent experimental measurements. 

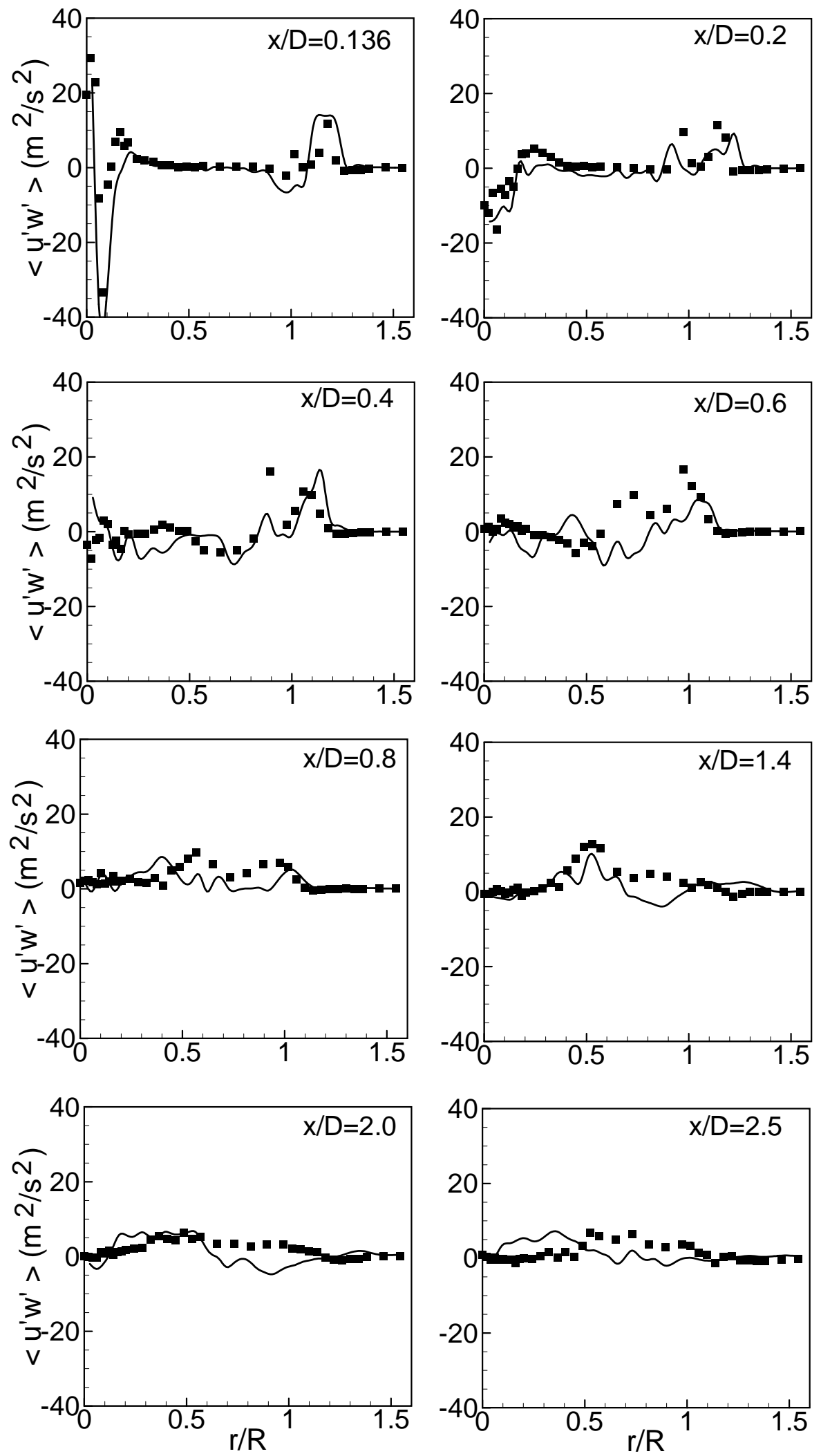

Figure 17: Comparison of Reynolds shear stress $\left\langle u^{\prime} w^{\prime}\right\rangle$. Lines represent LES results, and symbols represent experimental measurements. 


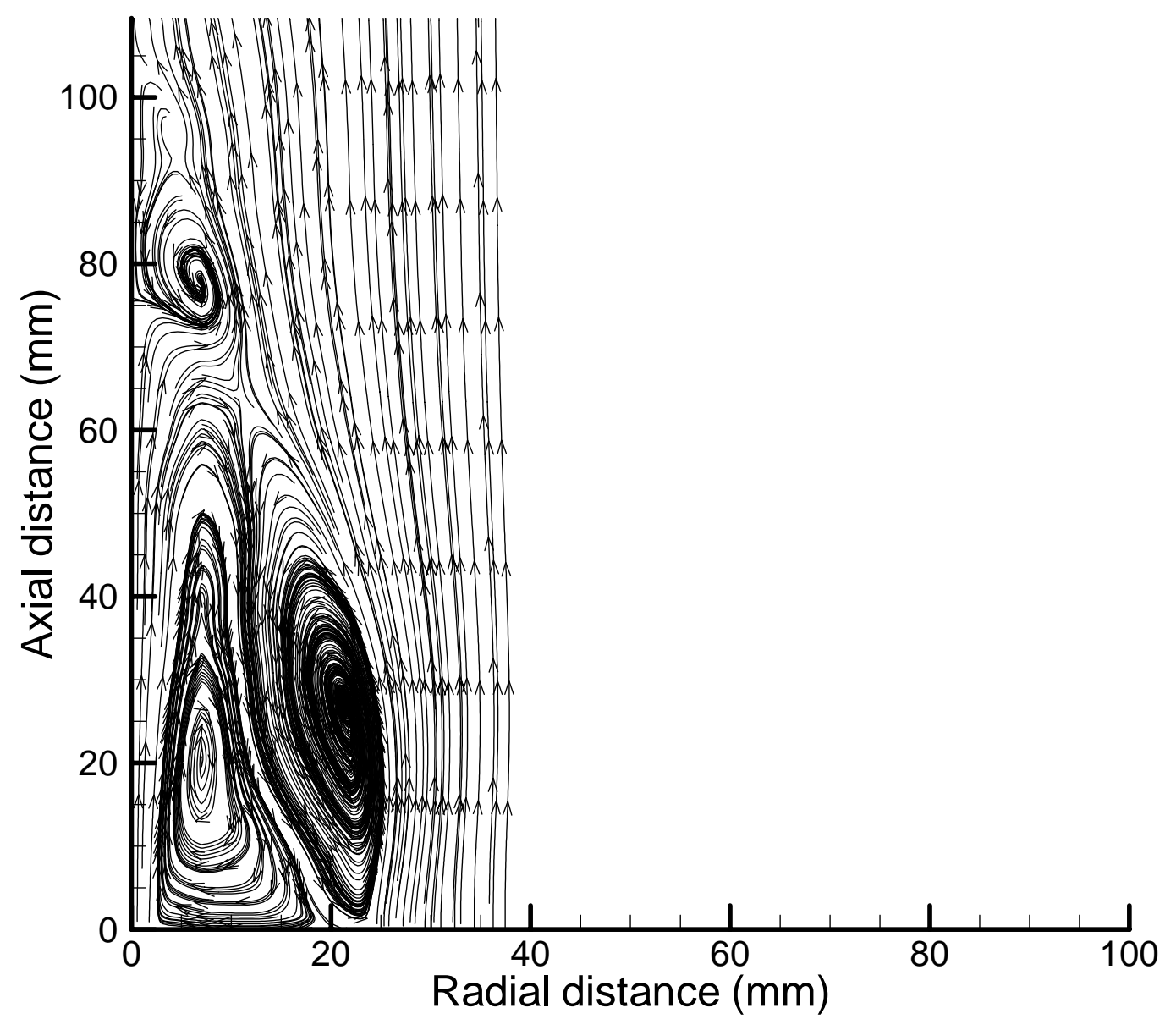

Figure 18: Streamlines generated from the mean velocity field of the high swirl case (N16S159) obtained from LES calculation. 

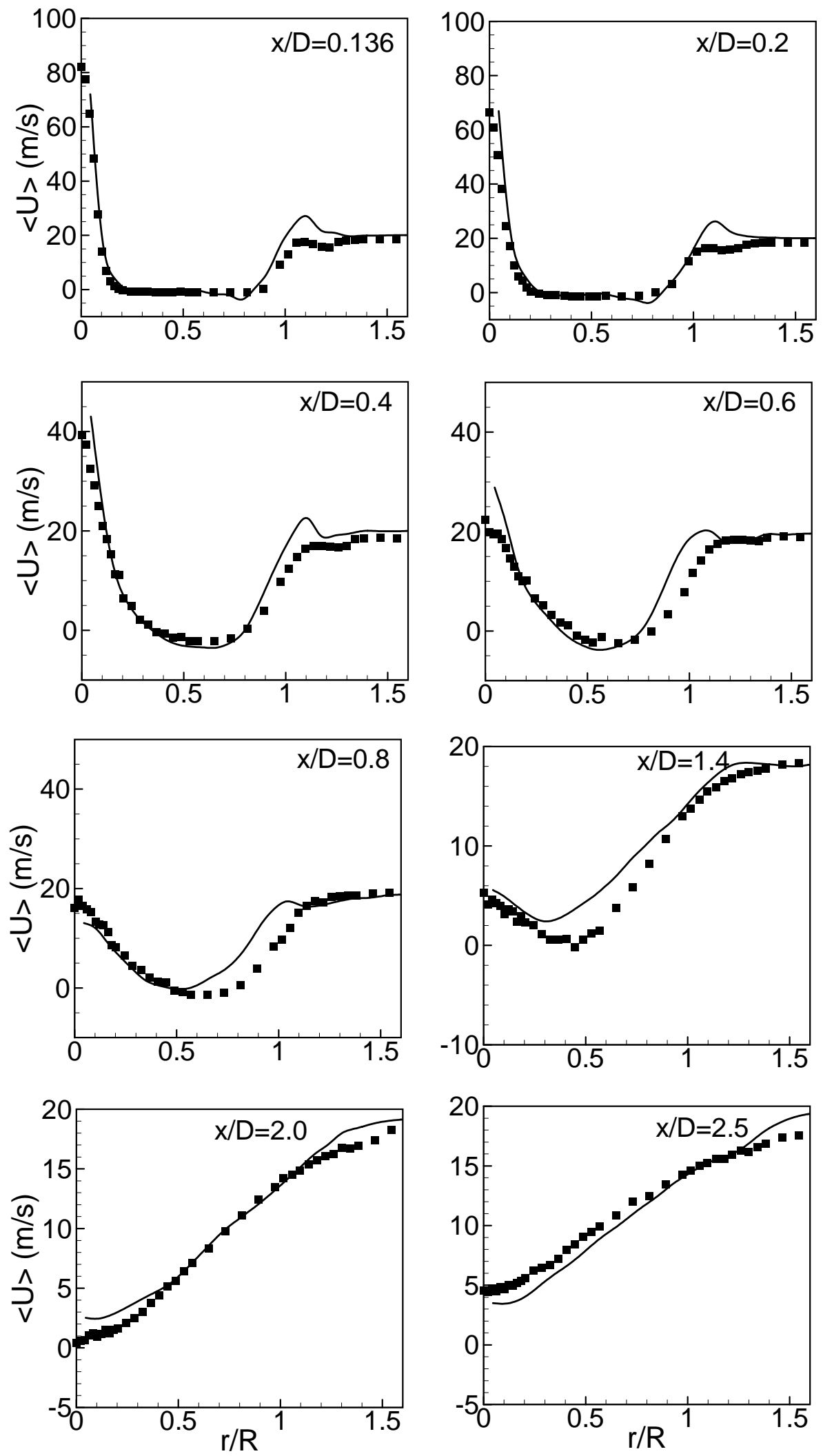

Figure 19: Comparison of mean axial velocity. Lines represent LES results, and symbols represent experimental measurements. 

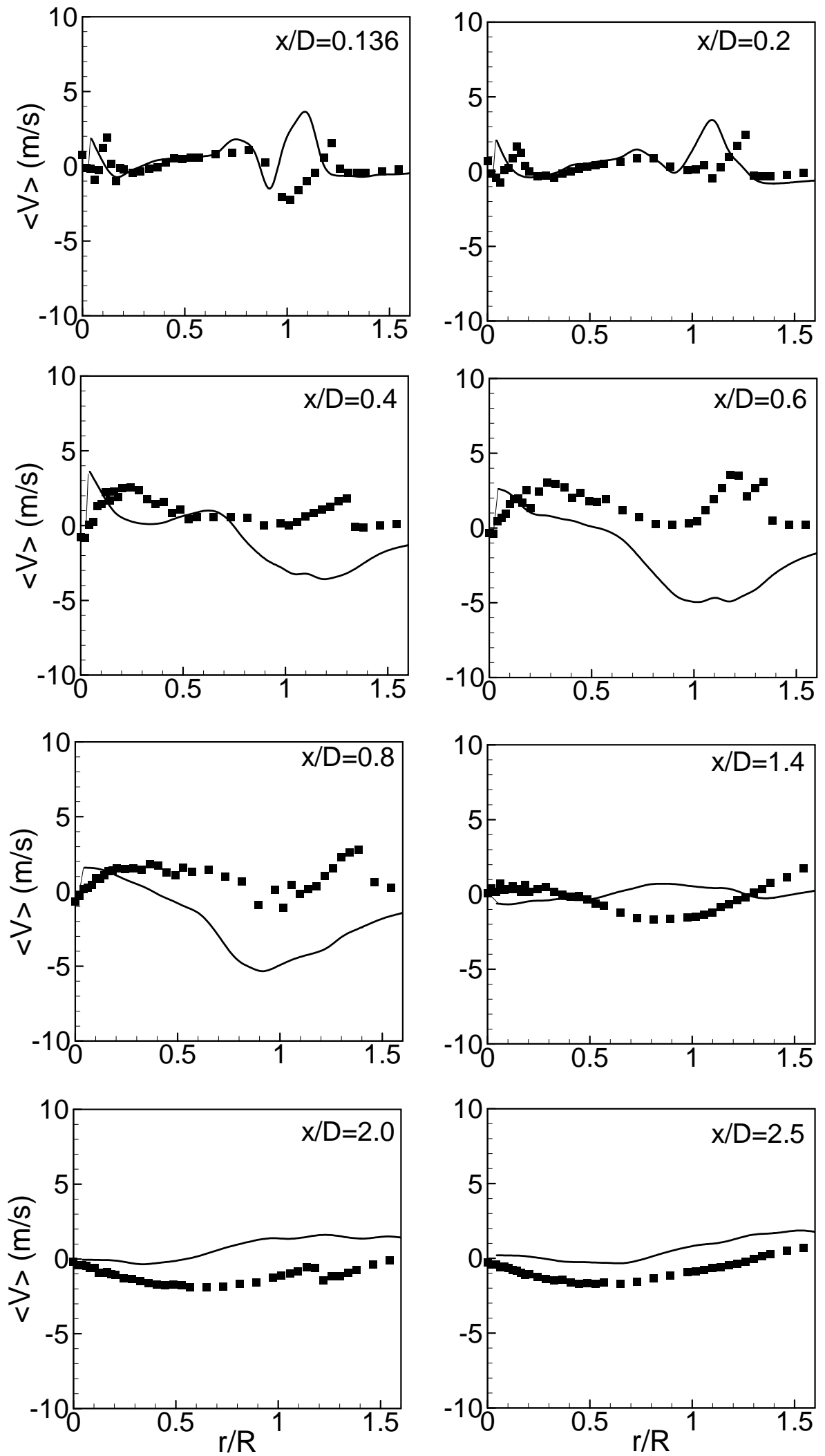

Figure 20: Comparison of mean radial velocity. Lines represent LES results, and symbols represent experimental measurements. 

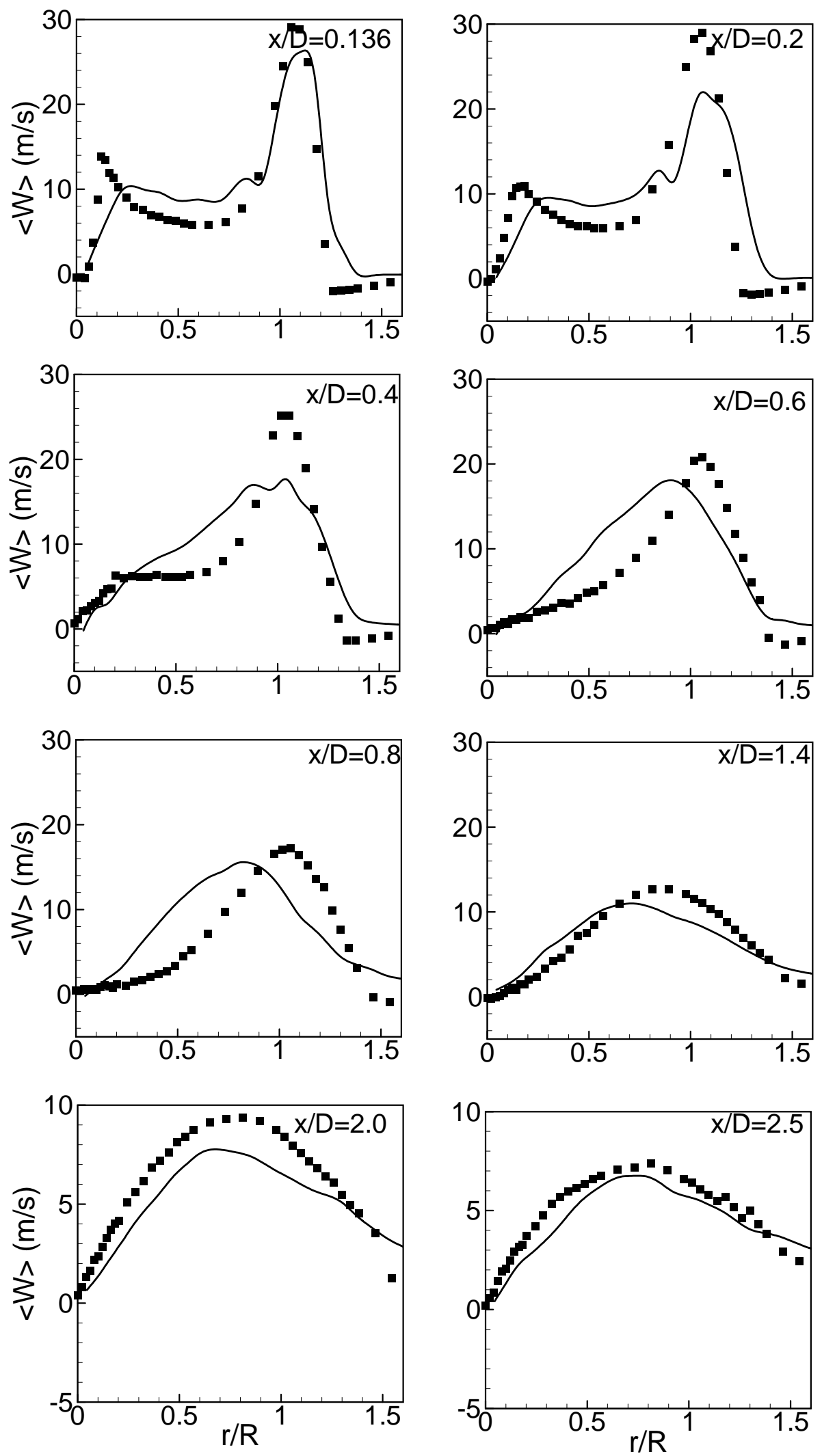

Figure 21: Comparison of mean swirl velocity. Lines represent LES results, and symbols represent experimental measurements. 

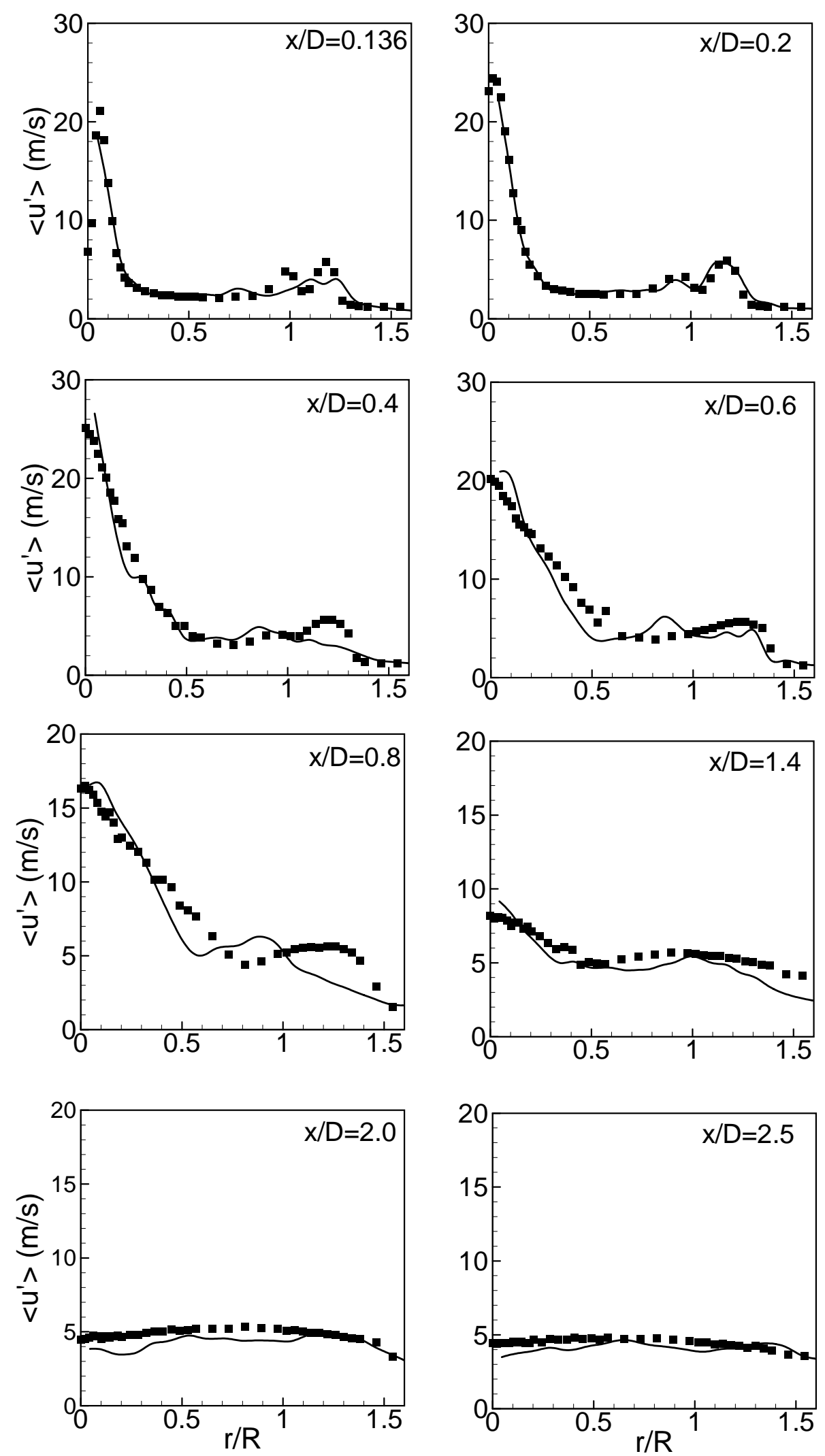

Figure 22: Comparison of rms of axial velocity fluctuations. Lines represent LES results, and symbols represent experimental measurements. 

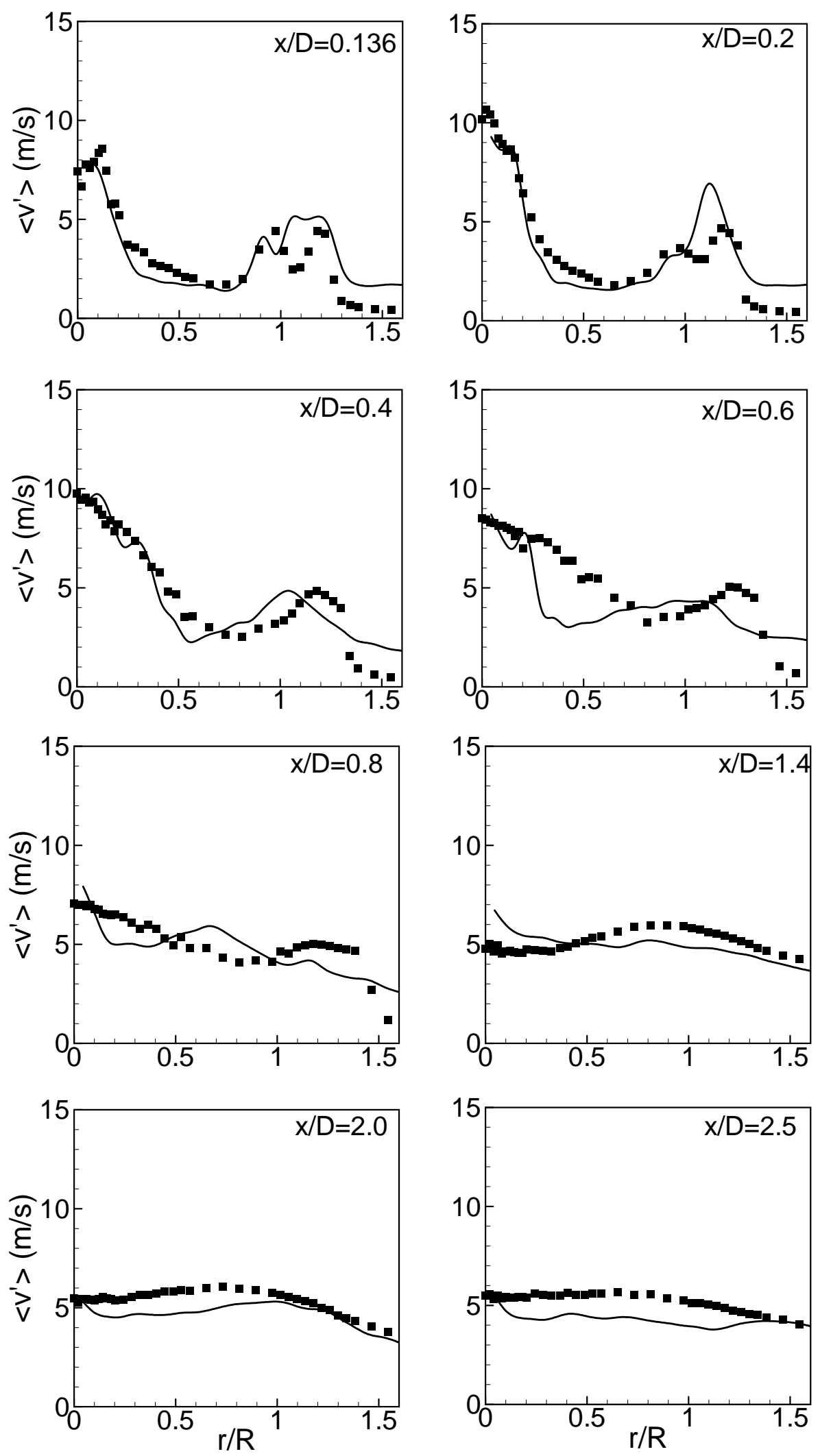

Figure 23: Comparison of rms of radial velocity fluctuations. Lines represent LES results, and symbols represent experimental measurements. 

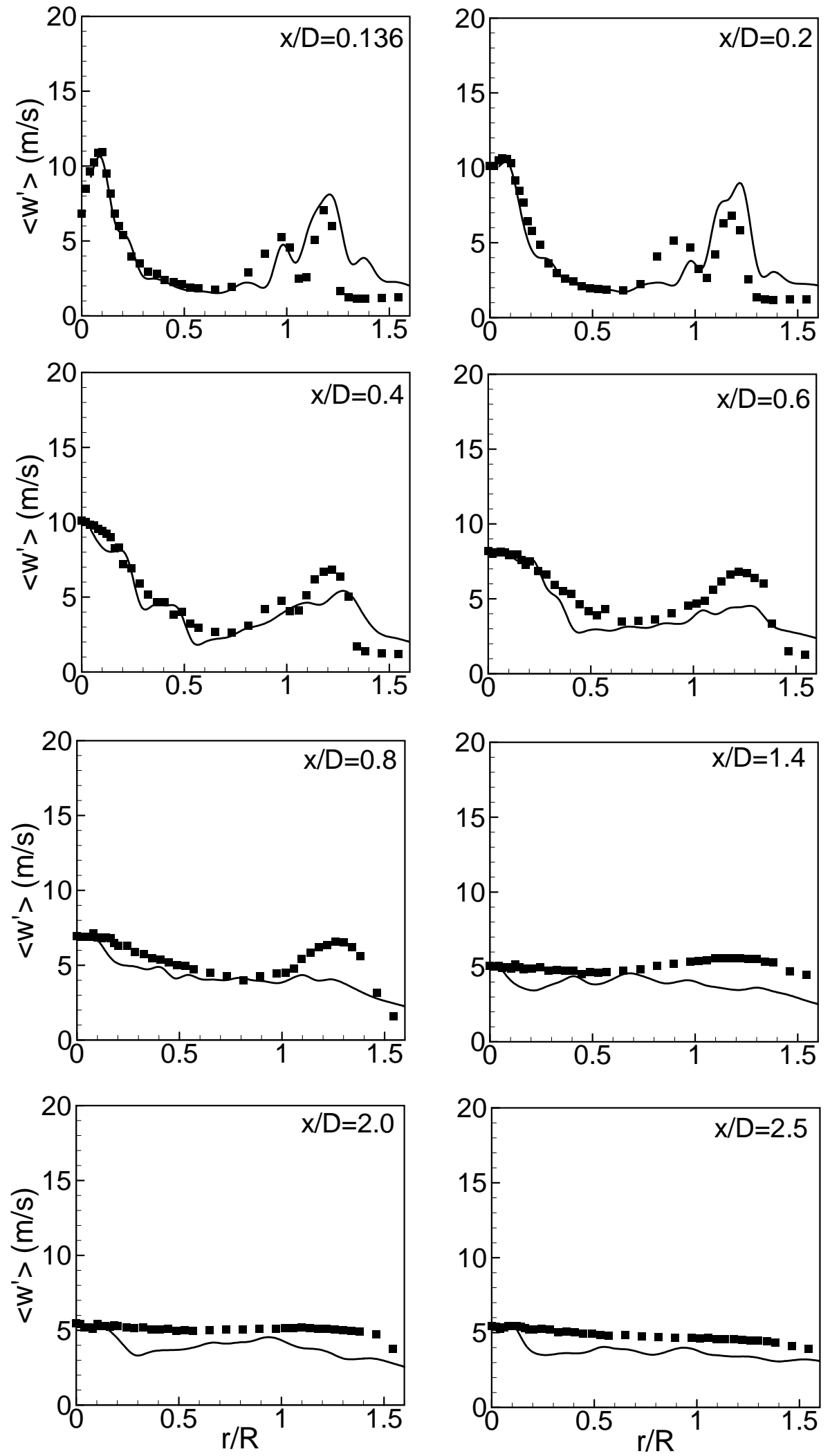

Figure 24: Comparison of rms of swirl velocity fluctuations. Lines represent LES results, and symbols represent experimental measurements. 

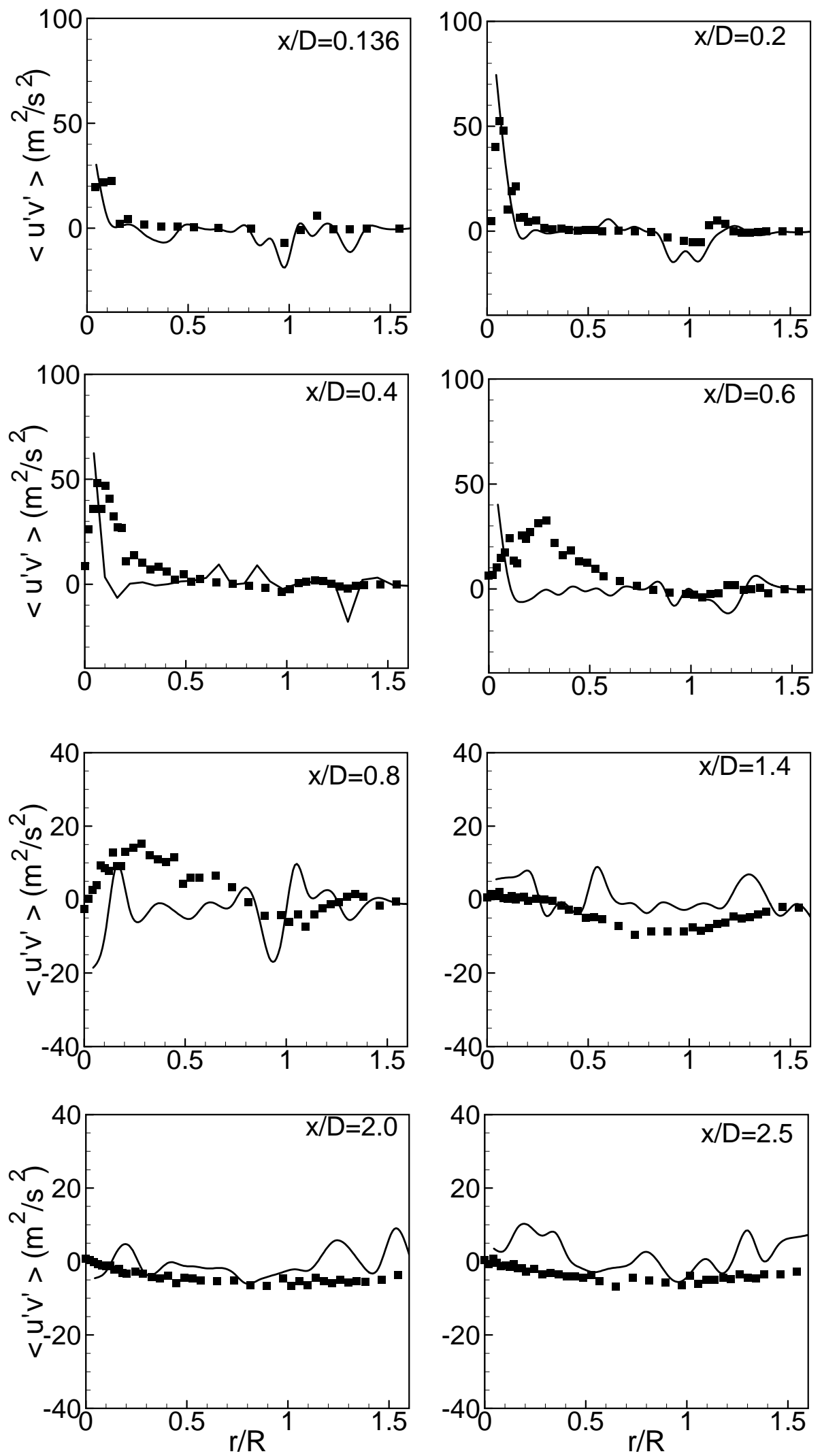

Figure 25: Comparison of Reynolds shear stress $\left\langle u^{\prime} v^{\prime}\right\rangle$. Lines represent LES results, and symbols represent experimental measurements. 

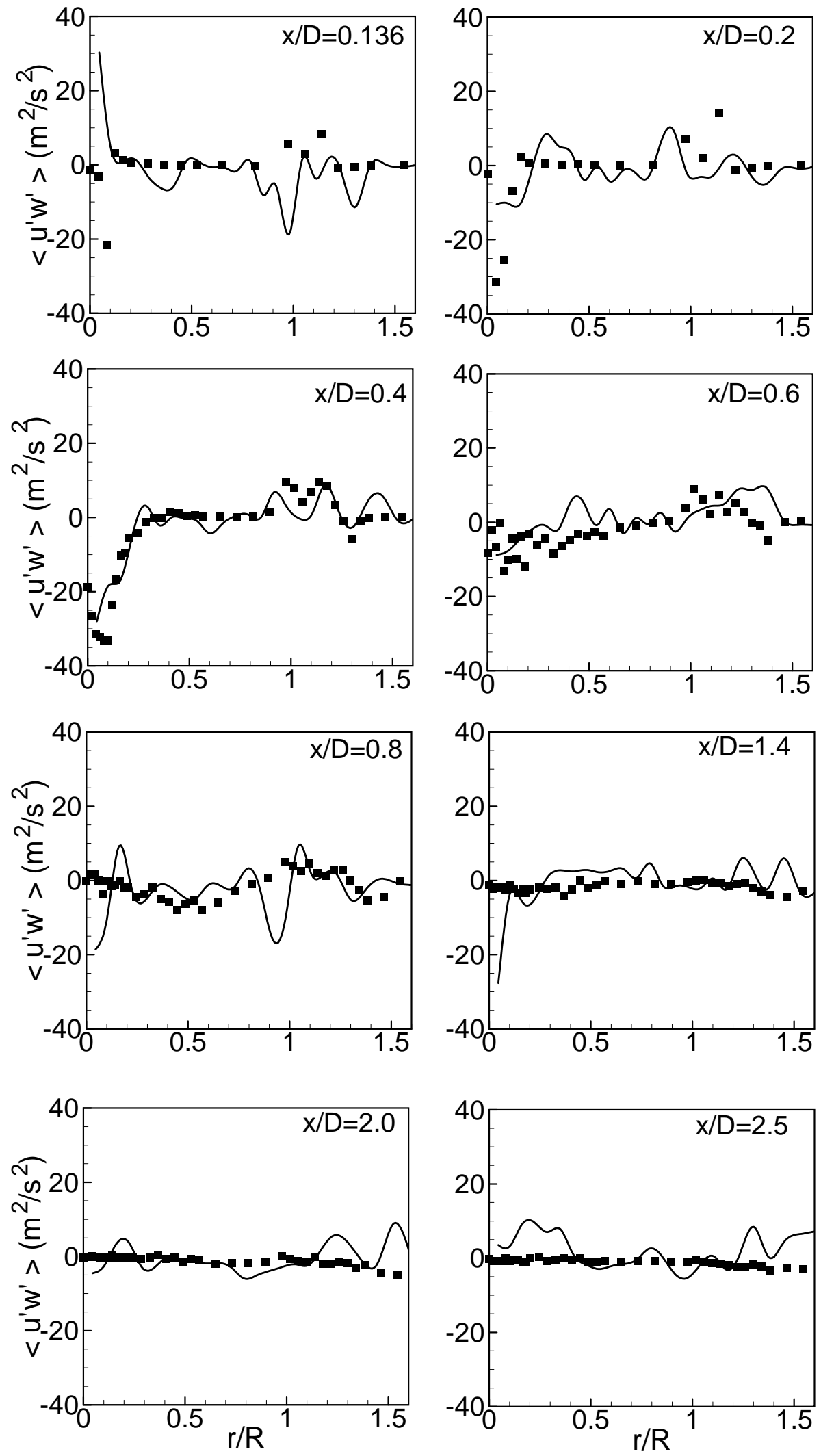

Figure 26: Comparison of Reynolds shear stress $\left\langle u^{\prime} w^{\prime}\right\rangle$. Lines represent LES results, and symbols represent experimental measurements. 